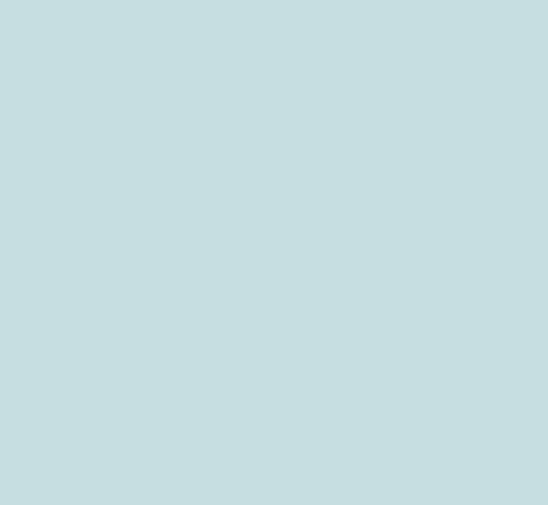

\title{
Luminescence-based Imaging Approaches in the Field of Interventional Molecular Imaging ${ }^{1}$
}

Fijs W. B. van Leeuwen, PhD

James C. H. Hardwick, MA, MB BChir, MRCP, PhD Arian R. van Erkel, MD, PhD
${ }^{1}$ From the Department of Radiology, Interventional Molecular Imaging Laboratory and Section of Interventional Radiology (F.W.B.v.L., A.R.v.E.), and Department of Gastroenterology (J.C.H.H.), Leiden University Medical Center, Albinusdreef 2, P0 Box 9600, 2300 RC Leiden, the Netherlands. Received November 24, 2013; revision requested January 15, 2014; revision received June 18; accepted August 11; final version accepted October 10. Supported in part by a Dutch Cancer Society translational research award (grant PGF 2009-4344) and an NWO-STW-VIDI grant (STW BGT 11272). Address correspondence to F.W.B.v.L. (e-mail: f.w.b.van_leeuwen@lumc.n).
Luminescence imaging-based guidance technologies are increasingly gaining interest within surgical and radiologic disciplines. Their promise to help visualize molecular features of disease in real time and with microscopic detail is considered desirable. Integrating luminescence imaging with three-dimensional radiologic- and/or nuclear medicine-based preinterventional imaging may overcome limitations such as the limited tissue penetration of luminescence signals. At the same time, the beneficial features of luminescence imaging may be used to complement the routinely used radiologic- and nuclear medicine-based modalities. To fully exploit this integrated concept, and to relate the largely experimental luminesce-based guidance approaches into perspective with routine imaging approaches, it is essential to understand the advantages and limitations of this relatively new modality. By providing an overview of the available luminescence technologies and the various clinically evaluated exogenous luminescent tracers (fluorescent, hybrid, and theranostic tracers), this review attempts to place luminescence-based interventional molecular imaging technologies into perspective to the available radiologic- and/or nuclear medicine-based imaging technologies. At the same time, the transition from anatomic to physiologic and even molecular interventional luminescence imaging is illustrated.

${ }^{\circ}$ RSNA, 2015

Online supplemental material is available for this article. 
maging technologies are increasingly being used to provide guidance prior to and during interventions $(1,2)$. While the use of anatomic imaging information during radiologic or surgical interventions is a common practice, it is desirable to obtain a real-time readout of the target lesion, preferably one that includes specific molecular parameters. This feature may be used to ensure a more effective treatment, while minimizing the amount of healthy tissue that is damaged. In the clinic, luminescence imaging refers to the visualization of lesions by using either light emitted from tissue (so-called tissue autofluorescence [a fluorescent signal produced by

\section{Essentials}

- A basic understanding of the technique of luminescence-based imaging approaches is required before radiologists, nuclear medicine physicians, and surgeons can incorporate this approach in their spectrum of imaging modalities.

- Preinterventional staging with established total body and/or cross-sectional imaging technologies is mandatory to allow minimally invasive luminescencebased guidance to deeper-lying lesions or to lesions with an unknown location.

- The beneficial features of luminescence imaging may be used to complement more established radiologic- and nuclear medicine-based imaging approaches.

- Despite the theoretical advantages of near-infrared fluorescence imaging, luminescent tracers that emit within the visual spectrum have been most widely applied in clinical trials.

- Various clinical trials have illustrated that luminescent tracers may ease lesion identification for, for example, surgeons, but for most of these procedures the effect on the clinical outcome still has to be proven. endogenous molecules, eg, hemoglobin]) or a particular luminescent tracer (exogenous contrast agent [either fluorescent, hybrid, or Cerenkov]). Clinically, luminescence-based imaging was first introduced in 1924 (3) with the observation of a "red" fluorescent emission coming from hematoporphyrin illuminated with a Woods lamp. In the 1940s, the fluorescent dye fluorescein was shown to allow delineation of brain tumors (4). In the past decade, the development of light-sensitive (near-infrared) fluorescence cameras has initiated a renewed interest in these optical technologies. The main driving force behind this revival is the theoretical potential to improve realtime (microscopic) identification and delineation of lesions that cannot be accurately defined otherwise, for example, by palpation and/or the naked human eye. However, before radiologists, nuclear medicine physicians, and surgeons can incorporate such luminescence-based imaging approaches in their spectrum of imaging modalities, they will have to acquire a basic understanding of this modality.

Since luminescence-based imaging is limited by its signal penetration $(<$ $1 \mathrm{~cm}$ ), the utility of this technology is driven by the accessibility of the tissue with a camera. This means the technology can only be used to identify superficial lesions with a known origin, for example, colorectal neoplasia (5). The use of luminescence-based imaging for deeper-lying lesions or lesions with an unknown location still requires the simultaneous or complementary use of established total body and/or cross-sectional imaging technologies such as ultrasonography (US), x-raybased technologies, computed tomography (CT), magnetic resonance (MR) imaging, single photon emission CT (SPECT), or positron emission tomography (PET) (Fig 1). These wellestablished radiologic and/or nuclear medicine modalities not only provide a means of "preselecting" patients who may benefit from luminescence imaging, they may also serve as a threedimensional road map or even provide a basis for mixed-reality navigation, followed by local luminescence imaging (6-8). Ultimately, patients may benefit from an integrated approach wherein routine clinical imaging tools are complemented with luminescence imaging (9). In this concept, the latter makes it possible to zoom in on molecular features of disease spread (10). When such integration is achieved, a so-called combined pre-intervention and interventional imaging paradigm is created.

Two main principles describe the essence of this paradigm: (a) use of preintervention (molecular) imaging data to guide interventional luminescence imaging and (b) use of interventional luminescence-based imaging to help obtain more detailed (molecular) information from the target lesion(s). These two forms of complementary imaging may be achieved by using either two separate but complementary tracers, of which one is luminescent in nature, or by using hybrid tracers that can be detected by using multiple different imaging modalities.

This review attempts to place the role of clinically applied luminescent tracers and luminescence-based optical imaging technologies into perspective. Only exogenous tracers (fluorescent, hybrid, and theranostic) that have been described in clinical trials are reviewed (Tables 1-3). Of these concepts, the underlying physics, shortcomings, overlap with findings with use of radiologic and/or nuclear medicine modalities, and the potential added value of luminescencebased optical imaging technologies are

Published online

10.1148/radiol.2015132698 Content code: MI

Radiology 2015; 276:12-29

\section{Abbreviations:}

CEA $=$ carcinoembryonic antigen

EGFR = epidermal growth factor receptor

$\mathrm{FDG}=$ fluorodeoxyglucose

FITC = fluorescein isothiocyanate

$I C G=$ indocyanine green

$\mathrm{mAB}=$ monoclonal antibody

$\mathrm{PDD}=$ photodynamic diagnosis

5-ALA $=5$-aminolevulinate

Conflicts of interest are listed at the end of this article. 


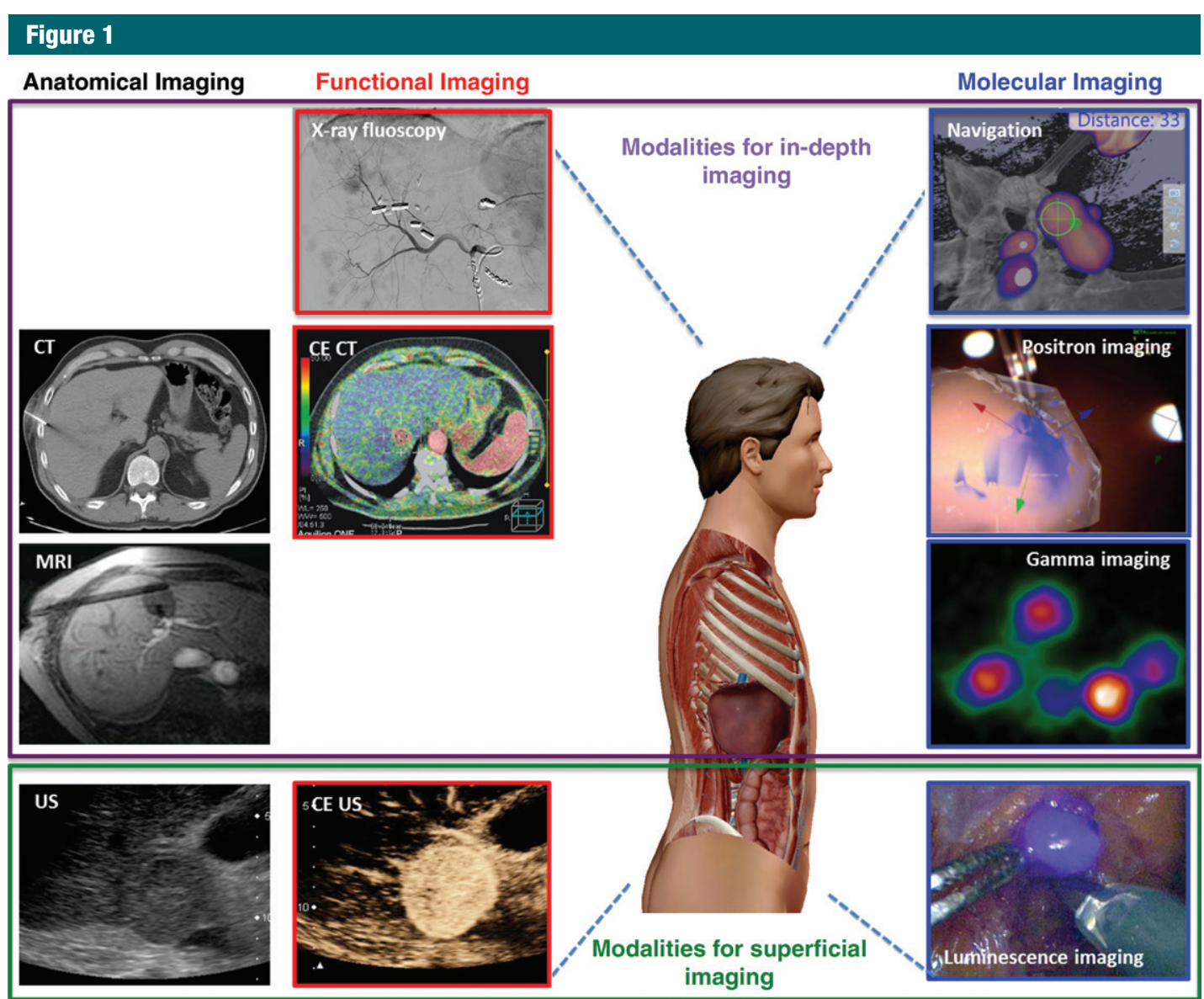

Figure 1: Schematic illustration of the different imaging technologies available for interventional guidance. Modalities are subdivided into anatomic, physiologic, and molecular groups. Note that molecular modalities may also be used to visualize physiologic parameters. To place luminescence imaging in context with the other available imaging technologies, a separation is made between true "in-depth" modalities and superficial technologies such as US $(<10 \mathrm{~cm})$ and luminescence imaging $(<1 \mathrm{~cm})$. Images are purely meant for illustration. The images on the left depict imaging the liver in a preinterventional (diagnostic imaging) setting. Images on the right show a range of applications that are mainly used for guidance during the intervention in the intraoperative setting. The anatomic image was made by using the visual body software package (Human Anatomy Atlas, version 3.0.1; Argosy, Newton Upper Falls, Mass). CE = contrast enhanced.

discussed. A particular focus is placed on the transition from anatomic imaging to physiologic and even molecular imaging approaches in the field of oncologic interventions.

\section{Basic Principle of Luminescence-based Imaging}

Essentially, luminescence-based imaging focuses on the detection of light that is emitted from a diseased area. The emission spectrum of luminescent tracers intended for medical use encompasses ultraviolet light $(<400 \mathrm{~nm})$, visible light $(400-650 \mathrm{~nm})$, far-red light (650-780 nm), and the international union of pure and applied chemistry defined near-infrared range of 780-2000 $\mathrm{nm}$ (11). This review discusses the use of two types of luminescent signals, namely fluorescence (used in fluorescent, hybrid, and theranostic tracers) and Cerenkov luminescence. The physical principles and limitations of these technologies are briefly introduced below.

\section{Fluorescence}

The basic principle of fluorescence imaging is that dyes convert light shined onto them (excitation light) into light of a longer wavelength (emission light); to get a light signal from a fluorescent tracer, light needs to go in first. For that reason, detection of fluorescent signals requires a modality comprising both an excitation light source and a light-sensitive camera that can detect the emitted light.

When the light spectrum used for excitation falls within the excitation spectrum of a specific fluorescent dye, the dye is converted to its excited state (Fig 2, A). When this dye decays back to its stable ground-state, light with a lower energy is emitted (Fig 2, B). Both the excitation and emission 


\section{Table 1}

\section{Clinically Evaluated Fluorescent Tracers}

\begin{tabular}{|c|c|c|c|c|c|}
\hline Fluorescent Tracer & $\begin{array}{l}\text { Regulatory } \\
\text { Status (only FDA } \\
\text { mentioned) }\end{array}$ & Clinical Indication Studied ${ }^{*}$ & $\lambda_{\text {ex max }} / \lambda_{\text {em max }}(n m)$ & $\begin{array}{l}\text { Quantum } \\
\text { Yield (\%) }\end{array}$ & $\begin{array}{l}\text { Molar Absorbance } \\
\text { Coefficient } \\
\left(\mathrm{mol}^{-1} \mathrm{~cm}^{-1}\right)\end{array}$ \\
\hline \multicolumn{6}{|l|}{ Nontargeted } \\
\hline \multirow[t]{5}{*}{ Fluorescein } & $\begin{array}{l}\text { (NDA)022186 \& } \\
\text { (NDA)021980 }\end{array}$ & $\begin{array}{l}\text { Angiography of, for example, the eye (reference } \\
\text { standard) and epicerebral microcirculation } \\
(n=5)(42,107)\end{array}$ & $488 / 515$ & $92(108)$ & $8 \times 10^{4}(108)$ \\
\hline & & Ureter visualization $(n=12)(54)$ & & & \\
\hline & & Lymphangiography $(n=>1000)(56)$ & & & \\
\hline & & $\begin{array}{l}\text { String test for gastrointestinal hemorrhage }(n=32) \\
\text { (109) }\end{array}$ & & & \\
\hline & & Confocal endomicroscopy of colon $(n=42)(71)$ & & & \\
\hline FITC-dextran 150000 & & Lymphangiography ( $n=39)(57)$ & $488 / 515$ & $92(108)$ & $8 \times 10^{4}(108)$ \\
\hline Cresyl violet & & Confocal endomicroscopy of colon $(n=36)(72)$ & $591 / 628$ & $54(110)$ & - \\
\hline Acrilflavine hydrochloride & & Confocal endomicroscopy of colon $(n=8)(70)$ & $416 / 514$ & - & - \\
\hline \multirow[t]{3}{*}{ Methylene blue } & With FDA warning & Ureter visualization $(n=12)(55)$ & $670 / 680$ & $4(111)$ & $9 \times 10^{4}(111)$ \\
\hline & & Parathyroid adenoma imaging $(n=12)(49)$ & & & \\
\hline & & $\begin{array}{l}\text { Melanoma delineation via fluorescence polarization } \\
\text { imaging (ex vivo, } n=6)(112)\end{array}$ & & & \\
\hline Omocyanine & & Breast cancer detection $(n=11)(46)$ & $761 / 781$ & $17(46)$ & - \\
\hline \multirow[t]{9}{*}{ ICG } & $\begin{array}{l}\text { (ANDA) } 040811 \& \\
\text { (NDA) } 011525\end{array}$ & $\begin{array}{l}\text { Perfusion of, for example, kidney }(n=94) \text { or free-flap } \\
\quad \text { reconstruction }(n=20)(40,113)\end{array}$ & $780 / 820$ & $3.3(41)$ & $4 \times 10^{5}(114)$ \\
\hline & & Angiography of, for example, the eye (1000 eyes) (115) & & & \\
\hline & & Lymphangiography $(n=22)(116)$ & & & \\
\hline & & Uretereral stenosis $(n=7)(117)$ & & & \\
\hline & & $\begin{array}{l}\text { Tumor visualization of, for example, HCC or colorectal } \\
\text { liver metastasis }(n=49)(104)\end{array}$ & & & \\
\hline & & Cholangiography $(n=13)(50)$ & & & \\
\hline & & Hepatic segmentectomy(118) & & & \\
\hline & & Confocal mini-laparoscopy of liver $(n=22)(29)$ & & & \\
\hline & & Sentinel node mapping $(n=18)(58)$ & & & \\
\hline \multicolumn{6}{|l|}{ Targeted } \\
\hline Folate-FITC & & Folate receptor $\alpha^{+}$ovarian carcinoma $(n=10)(75)$ & $488 / 515$ & $92(108)$ & $8 \times 10^{4}(108)$ \\
\hline CEA-mAB-FITC & & $\begin{array}{l}\text { Colorectal carcinoma imaging ( } n=1 \text {; ex vivo, } n=5 \text { ) } \\
\text { (119) }\end{array}$ & $488 / 515$ & $92(108)$ & $8 \times 10^{4}(108)$ \\
\hline VRPMLQ-FITC & & Colonic dysplasia $(n=26)(79)$ & $488 / 515$ & $92(108)$ & $8 \times 10^{4}(108)$ \\
\hline ASYNYDA-GGGSK-FITC & & Esophageal neoplasia $(n=25)(80)$ & $488 / 515$ & $92(108)$ & $8 \times 10^{4}(108)$ \\
\hline GE137 & & c-MET-overexpressing colon lesions $(n=15)(5)$ & $650 / 670$ & 28 & $2 \times 10^{5}(120)$ \\
\hline GGT-HMRG & & $\gamma$-GGT (ex vivo) (81) & $501 / 524$ & $81(121)$ & $6 \times 10^{4}(121)$ \\
\hline $\begin{array}{l}\text { anti-EGFR mAB Alexa } \\
\quad \text { Fluor } 488\end{array}$ & & Colorectal neoplasia $(n=37)(77)$ & $488 / 520$ & 92 & $7 \times 10^{4}$ \\
\hline MG7-AF488 & & Gastric cancer (ex vivo, 46 samples) (122) & $488 / 520$ & 92 & $7 \times 10^{4}$ \\
\hline CW800-bevacizumab & & $\begin{array}{l}\text { VEGF (breast cancer, } n=20 \text { [no in vivo data } \\
\text { published yet]) (123) }\end{array}$ & $783 / 801$ & $12(124)$ & $2 \times 10^{5}(125)$ \\
\hline
\end{tabular}

Note.-FDA $=$ Food and Drug Administration, $\lambda_{\mathrm{ex} \max }=$ maximum peak excitation wavelength, $\lambda_{\text {em } \max }=$ maximum peak emission wavelength, $\mathrm{FITC}=$ fluorescein isothiocyanate, ICG $=$ indocyanine green, $\mathrm{HCC}=$ hepatocellular carcinoma, $\mathrm{CEA}=$ carcinoembryonic antigen, $\mathrm{mAB}=$ monoclonal antibody, $\mathrm{GGT}=$ glutamyltranspeptidase, EGFR $=$ epidermal growth factor receptor, VEGF $=$ vascular endothelial growth factor.

* The number of patients is from representative studies; the clinical use of the compounds is not necessarily limited to these studies.

spectra dictating this process have a range and a maximum peak (maximum peak excitation wavelength, or $\lambda_{\text {ex max }}$, and maximum peak emission wavelength, or $\lambda_{\mathrm{em} \max }$ ) that is characteristic for individual fluorescent dyes (Tables 1-3), meaning camera settings have to be tailored toward these spectra depending on the fluorescent tracer used. For accurate detection of the signal, the eye or camera also needs to have a good sensitivity in the 


\section{Table 2}

\section{Hybrid Tracers Used for Image-guided Surgery}

\begin{tabular}{|c|c|c|c|c|c|}
\hline Hybrid Tracer & $\begin{array}{l}\text { Regulatory } \\
\text { Status (only FDA } \\
\text { mentioned) }\end{array}$ & Clinical Indications Studied* & $\lambda_{\text {ex max }} / \lambda_{\text {em max }}(n m)$ & $\begin{array}{l}\text { Quantum } \\
\text { Yield (\%) }\end{array}$ & $\begin{array}{l}\text { Molar Absorbance } \\
\text { Coefficient } \\
\left(\mathrm{I} \mathrm{mol}^{-1} \mathrm{~cm}^{-1}\right)\end{array}$ \\
\hline \multirow{3}{*}{$\begin{array}{l}\text { Ionidated methylene blue } \\
\quad\left({ }^{125}\left|{ }^{131}\right|, \text { or }{ }^{123} \mid\right)\end{array}$} & & Sentinel node mapping $(n=12)(88)$ & $670 / 680$ & $4(111)$ & $9 \times 10^{4}(111)$ \\
\hline & & Parathyroid imaging $(n=5)(86)$ & & & \\
\hline & & Melanoma metastases $(n=6)(87)$ & & & \\
\hline${ }^{131}$-diiiodofluorescein & & Brain tumors $(n=12)(82)$ & $488 / 515$ & $92(108)$ & $8 \times 10^{4}(108)$ \\
\hline ICG-99mTC-nanocolloid & & Sentinel node identification $(n=11)(13)$ & $780 / 820$ & $3.3(44)$ & $4 \times 10^{5}(114)$ \\
\hline${ }^{18} \mathrm{~F}-\mathrm{FDG}$ & (NDA)021870 & Lymph node imaging $(n=4)(32)$ & $<200 /-$ & - & - \\
\hline 131 & (NDA)010929 & Thyroid $(n=1)(31)$ & $<200 /-$ & - & - \\
\hline
\end{tabular}

Note. - FDA $=$ Food and Drug Administration, $\lambda_{\text {ex max }}=$ maximum peak excitation wavelength, $\lambda_{\text {em max }}=$ maximum peak emission wavelength, ${ }^{125} \mathrm{I}=$ iodine $125,{ }^{131} \mathrm{I}=$ iodine $131,{ }^{123} \mathrm{I}=$ iodine 123 , ${ }^{99 m} \mathrm{Tc}=$ technetium Tc $99 \mathrm{~m},{ }^{18} \mathrm{~F}=$ fluoride $18, \mathrm{FDG}=$ fluorodeoxyglucose.

* The number of patients mentioned is from representative studies; the clinical use of the compounds is not necessarily limited to these studies.

\section{Table 3}

\section{Theranostic Tracers}

\begin{tabular}{|c|c|c|c|c|c|}
\hline Theranostic Tracer & $\begin{array}{l}\text { Regulatory } \\
\text { Status (only FDA } \\
\text { mentioned) }\end{array}$ & Clinical Indications Studied ${ }^{*}$ & $\lambda_{\text {ex max }} / \lambda_{\text {em max }}(n m)$ & $\begin{array}{l}\text { Quantum } \\
\text { Yield (\%) }\end{array}$ & $\begin{array}{l}\text { Molar Absorbance } \\
\text { Coefficient } \\
\left(\mathrm{I} \mathrm{mol}^{-1} \mathrm{~cm}^{-1}\right)\end{array}$ \\
\hline Doxycycline & $\begin{array}{l}\text { (ANDA)062569 \& } \\
\text { (ANDA)091406 } \\
\text { (intravenous injection) }\end{array}$ & Osteonecrosis $(n=2)(95)(n=15)(126)$ & $345 / 570$ & $5(127)$ & $1 \times 10^{4}(127)$ \\
\hline \multirow[t]{3}{*}{ Tetracyclin } & $\begin{array}{l}\text { (ANDA)061837 } \\
\quad \text { (oral administration) }\end{array}$ & Osteoid osteoma $(n=9)(128)$ & $357 / 570$ & $0.2(127)$ & $1 \times 10^{4}(127)$ \\
\hline & & $\begin{array}{l}\text { Nonmelanoma delineation via fluorescence } \\
\text { polarization imaging (ex vivo, } n=32 \text { ) (129) }\end{array}$ & & & \\
\hline & & $\begin{array}{l}\text { Melanoma delineation via fluorescence polarization } \\
\text { imaging (ex vivo, } n=6)(112)\end{array}$ & & & \\
\hline Demeclocycline & & $\begin{array}{l}\text { Nonmelanoma delineation via fluorescence } \\
\text { polarization imaging (ex vivo, } n=54)(129)\end{array}$ & $367 / 585$ & $4(127)$ & $1 \times 10^{4}(127)$ \\
\hline 5-ALA, HAL, MAL & & $\begin{array}{l}\text { PpIX-based PDD of a variety of malignancies } \\
\text { (eg, brain, bladder and oral cancer)(97) }\end{array}$ & $406 / 632$ & $1.1(130)$ & $3 \times 10^{4}(131)$ \\
\hline Hypericin & & $\begin{array}{l}\text { Wide application in PDD of, for example, bladder } \\
\text { cancer(103) }\end{array}$ & $595 / 600$ & 2 & $4 \times 10^{4}(132)$ \\
\hline mTHPC & & $\begin{array}{l}\text { Wide application in PDD of, for example, brain } \\
\text { tumors(102) }\end{array}$ & $417 / 650-653$ & $8.9(133)$ & $\begin{array}{l}2 \times 10^{5}(417 \mathrm{~nm} \\
[134]) \text { and } 3 \times 10^{4} \\
(650 \mathrm{~nm}[133])\end{array}$ \\
\hline
\end{tabular}

Note.-FDA $=$ Food and Drug Administration, $\lambda_{\text {ex } \max }=$ maximum peak excitation wavelength, $\lambda_{\text {em } \max }=$ maximum peak emission wavelength, PpIX $=$ protoporphyrin IX, 5-ALA $=5$-aminolevulinate, MAL $=$ methyl aminolevulinate, $\mathrm{HAL}=$ hexyl aminolevulinate, $\mathrm{mTHPC}=$ meta-tetrahydroxyphenylchlorin.

* The number of patients mentioned is from representative studies; the clinical use of the compounds is not necessarily limited to these studies.

range of the emitted light. To improve the specificity of cameras for emission light detection, often band-pass filters are used that block background signals such as reflected excitation light, autofluorescence, and ambient light. This latter technique can also be used to differentiate between various fluorescent signals (exemplified in Fig $3, D)$.

The signal intensity (photon flux) varies between fluorescent dyes and depends on: (a) the intensity of the excitation light (increasing the intensity of the light source will increase the amount of emitted photons); (b) the efficiency of light absorption (the absorption coefficient $[\varepsilon]$ is generally determined at the absorption maximum) (Tables 1-3); and (c) the quantum yield (the number of emitted photons per 


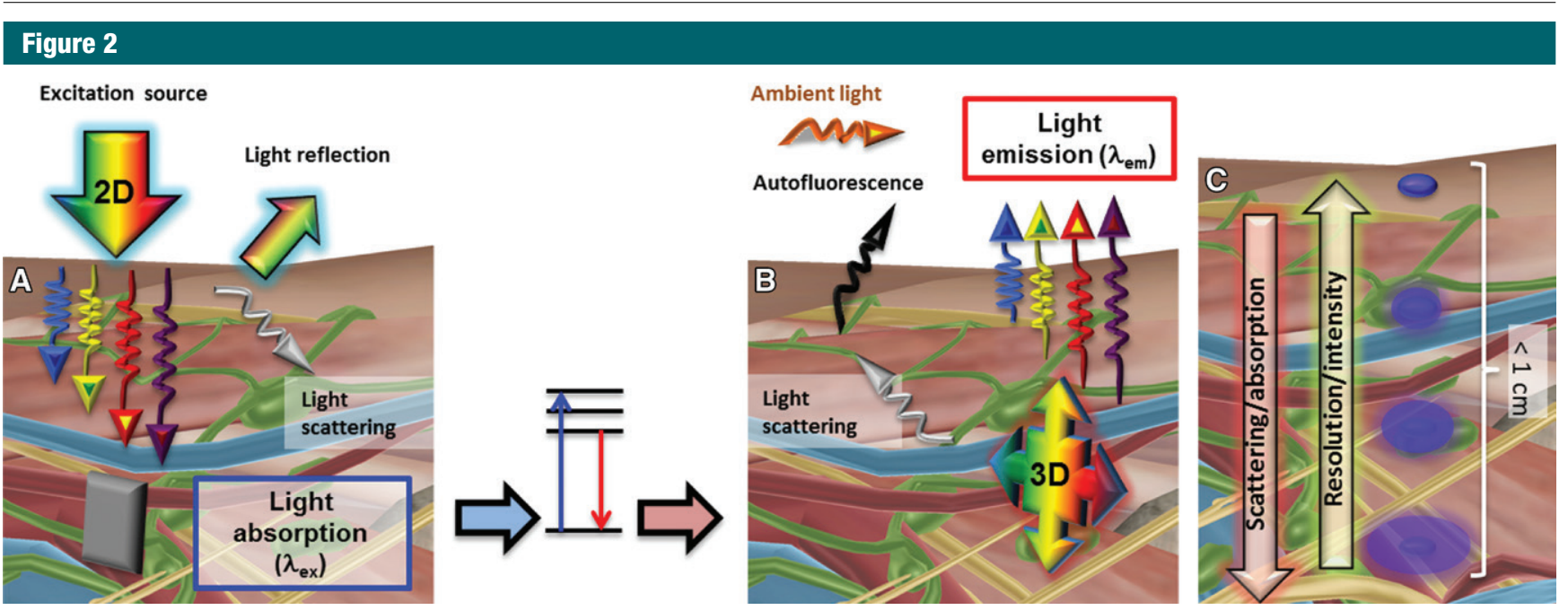

Figure 2: Schematic presentation of the different features influencing emission of luminescent signals in tissue. A, Excitation of a luminescent dye (only applicable for fluorescence). Penetration of excitation light is limited by reflection, scattering, and the efficacy of light absorption, leading to the excitation of the dye and subsequent relaxation. $B$, Emission of a luminescent signal (applicable to both fluorescence and Cerenkov). In addition to the effects mentioned in, $A$, detection of emitted light is further limited by presence of autofluorescence and ambient light. $C$, Due to the features described in, $A$ and $B$, luminescence imaging is a superficial technology, whereby the resolution is optimal at a minimal depth. This is illustrated by the clarity with which the blue signal is presented. Anatomic images were made by using the visual body software package (Human Anatomy Atlas, version 3.0.1; Argosy). $2 D=$ Two-dimensional, $3 D=$ three-dimensional, $\lambda_{e x}=$ peak excitation wavelength, $\lambda_{e m}=$ peak emission wavelength.

absorbed photon with a maximum of 100\%; Tables 1-3) (1).

\section{Cerenkov Luminescence}

Cerenkov luminescence is a "spontaneous light emission” generated by a $\beta$ particle traveling through a medium. The wide range of commercially available PET tracers $\left(\beta^{+}\right.$particle emitters $)$ and therapeutic isotopes $\left(\beta^{-}\right.$particle emitters) can potentially be used for Cerenkov luminescence imaging. For all $\beta$ particle-emitting isotopes, the Cerenkov emission signal is identical, showing a maximum peak emission wavelength below $200 \mathrm{~nm}$, accompanied by an emission tail extending into the near-infrared region. As a result, other than with fluorescence imaging, where each type of fluorescent dye requires specific camera filter settings, only one type of Cerenkov luminescence camera system is required to detect the Cerenkov luminescence signal from various $\beta$ particle-emitting tracers.

The photon flux of Cerenkov luminescence depends on the amount of the radiotracer administered; the radioactive decay of the isotopes will reduce the luminescence signal intensity with time. The efficacy of medium polarization and subsequent light conversion process increases with increasing energy of the $\beta$ particle (1).

\section{Limiting Factors in Luminescence Imaging}

Identical to the radionuclide emission signal, luminescence signals are emitted in three dimensions. However, with the currently available clinical luminescence camera systems, the luminescent signal can only be detected in two dimensions, geometrically limiting the sensitivity of detection. Other factors that may influence the signal detectability are: (a) light absorption by the tissue (occurring during both excitation and emission); (b) light scattering (occurring during both excitation and emission); (c) tissue autofluorescence; and (d) background signals caused by reflection and ambient light (Fig 2, A). While fluorescence imaging suffers from the limitations related to both the excitation and emission light, for Cerenkov luminescence imaging, only those related to the emitted light apply. That said, the limited signal intensity (orders of magnitude lower than those obtained during fluorescence imaging) and the broad emission profile of Cerenkov emission means that this technique will be influenced more by the presence of ambient light and requires longer acquisition times (1).

The influence of signal attenuation (and autofluorescence) is strongest at lower wavelengths. Therefore, at identical conditions, and assuming an identical photon flux, the penetration depth of a fluorescent dye emitting in the visible spectrum (eg, fluorescein) is more limited compared with that of a dye emitting in the near-infrared window (eg, ICG) (12). However, despite the excellent results obtained in phantom setups, clinical studies indicate that even near-infrared dyes such as ICG only add value in superficial imaging applications; lesions more than $1 \mathrm{~cm}$ deep might be missed due to faint and diffuse tissue staining (Fig 2, C) (13). Efforts aiming at improving the effective depth at which luminescent tracers can be detected are mainly based on using complementary feedback provided via radioisotopes 


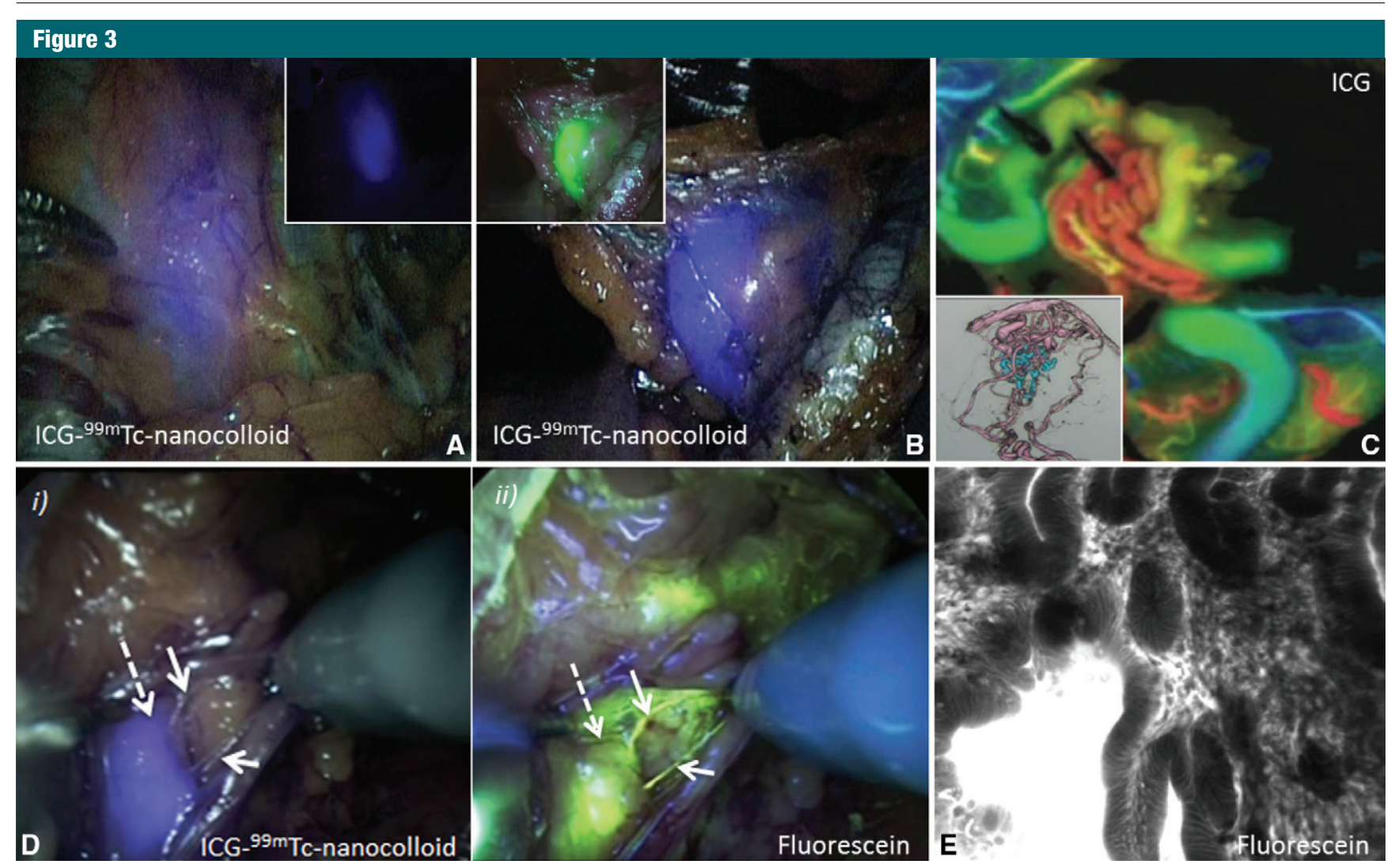

Figure 3: Achieved technologic advances to improve luminescence imaging. $A$, The availability of white light information helps place the luminescence findings in anatomic context. The image shows fluorescence imaging of the hybrid tracer ICG-99mTc-nancolloid resulting in the visualization of a prostate-draining sentinel node (fluorescence in blue). The insert shows the same image without the anatomic background detail. $B$, The pseudo-color used to depict the near-infrared fluorescence signal in a prostate-draining sentinel node (ICG-99mTc-nanocolloid) may affect the identification during surgery. Although the blue contrast (main image) is clear, the more contrast with the background is obtained the fluorescent signal is displayed in green (insert). C, Depiction of the fluorescence intensity of ICG in heat-map color coding can help depict vascular malformations in the brain. The insert shows corresponding three-dimensional rendering of a CT angiogram of the same lesion. (Reprinted, with permission, from reference 26.) D, Multiplexing allows sequential visualization of two different luminescent emissions that depict complementary features: i) a prostate-draining sentinel node containing ICG-99mTC-nanocolloid (fluorescence in blue; near-infrared settings) and ii) lymphatic vessels draining from the prostate to the sentinel node (fluorescence in yellow/green; fluorescein settings). Dotted arrow = the sentinel node, arrows = draining lymphatic ducts. $E$, Fiber-based microscopic fluorescence imaging can be used to visualize fluorescein accumulation at a cellular level in the lumen of the colon. The fluorescence image is shown as a black-and-white image; fluorescein is displayed in white. (Reprinted, with permission, from reference 35.)

(hybrid or Cerenkov tracers) and an alternative technology, namely photoacoustics, whereby the absorbance of light in a molecule leads to an excitation that can be detected with a US probe (14).

\section{Contrast Agent Sensitivity of Luminescence Imaging Modalities Relative to Radiologic- and Nuclear Medicine-based Imaging Modalities}

For interventional guidance, a variety of modalities is available, all of which have their own strengths and weaknesses. In particular, the contrast agent sensitivity, meaning the amount of moles of contrast agent required for detection, and thus the ability to depict molecular features of disease, strongly differs between these modalities (15).

Of the routinely used radiologic modalities, US has the highest contrast agent sensitivity $\left(10^{-9}\right.$ to $10^{-12}$ mol), but here the contrast agent mainly remains confined to the vascular system, limiting the use of this modality for molecular imaging purposes. The maximal contrast agent sensitivity of CT is approximately $10^{-3} \mathrm{~mol}$, for MR imaging it is $10^{-6}$ mol (pharmacological range). These low sensitivities restrict their ability to distinguish between biomarker expression levels. On the other hand, at nuclear medicine, PET and SPECT scanners can effectively depict tracer quantities of contrast material $\left(10^{-10}\right.$ to $10^{-15} \mathrm{~mol}$ ), allowing visualization of the molecular fingerprint of a disease (15). In theory, luminescence-based imaging modalities have a contrast agent sensitivity that is similar to that of US and nuclear medicine techniques $\left(10^{-9}\right.$ to $\left.10^{-12} \mathrm{~mol}\right)$, also enabling the visualization of molecular features (15). Here it should be noted that increasing the concentration of a 
fluorescent dye does not always lead to an increase in its fluorescent signal intensity; dye-dye interactions may result in a decreased emission signal, so-called self-quenching (16).

\section{Luminescence Imaging Systems}

Luminescence-based optical imaging can be applied in a number of different ways and has already been successfully integrated in endoscopic (eg, laparoscopy, colonoscopy, bronchoscopy) and open-surgery procedures. Herein, luminescence-based imaging can allow high-resolution identification of superficial lesions $(<1 \mathrm{~cm})$ (17). In general, the use of luminescent tracers has been driven by the commercial availability of luminescence imaging devices from established companies such as Olympus (Tokyo, Japan), Karl Storz GmbH \& Co KG (Tutlingen, Germany), Hamamatsu Photonics (Hamamatsu, Japan), Novadaq (Mississauga, Canada), and Intuitive Surgical (Sunnyvale, Calif). Many of these companies have a historic background in white light imaging and the visualization of luminescent tracers that emit light in the visible part of the spectrum (eg, protoporphyrin IX derivatives and fluorescein) or the visualization of tissue autofluorescence. Since the concept of luminescence imaging is the same across the light spectrum, a relatively straightforward modification of preexisting cameras now enables efficient detection of light in the near-infrared region of the light spectrum. The pace of these developments is stunning and the quality of the interventional images obtained has already reached full high-definition views, where the near-infrared fluorescent tracers used for angiography or sentinel node procedures can be accurately detected in real time and within the anatomic context of the specific patient (Fig 3). These efforts are further strengthened by an increasing number of small companies and research groups that are involved in the generation of alternative imaging devices; wellknown examples of camera systems for open-surgery procedures are the FLARE camera (the FLARE Foundation, Wayland, Mass) (18), the Fluoptics camera system (Fluoptics, Grenoble, France) (19), and the SurgVision camera (SurgVision, Heerenveen, the Netherlands) (20). Interesting to mention in this context is also the experimental hands-free goggles system from Liu et al $(21,22)$.

Microscopic optical imaging has been widely explored in ophthalmology (23) and in neurosurgery $(24,25)$. For these indications, the areas of interest are in a known and stable location of the body, and as a result, clinical fluorescence microscopes like those of Leica Camera (Nussloch, Germany) and Carl Zeiss (Jena, Germany) can be used. Although the name suggests otherwise, these fluorescence microscopes only provide a submillimeter resolution. Nevertheless, this submillimeter resolution was shown to enable the detection of malformations in small blood vessels, thereby improving, for example, the accuracy of clipping in patients with a cerebral aneurysm (26).

More recently there has been a drive toward the use of small fiberbased microscopic optical devices that can be applied during interventions. Commercially, modalities are available from, for example, Optiscan (Victoria, Australia), Mauna Kea Technologies (Paris, France), and Olympus. These systems explore fluorescence imaging to provide immediate microscopic feedback on the tissue histology and/ or morphology of lesions $(27,28)$, enabling so-called optical tissue biopsy. It should be mentioned that there is a long way to go before such technologies can replace conventional histologic evaluation. The image resolution that can be obtained ranges between 1 and $10 \mu \mathrm{m}$ (the diameter of a tumor cell is approximately $10 \mu \mathrm{m})$. While endocytoscopy only allows superficial imaging at this resolution, confocal laser endomicroscopy enables up to $250-\mu \mathrm{m}$ deep in-plane visualization (27) and confocal mini laparoscopy even reaches $350 \mu \mathrm{m}$ (29).

Research efforts to improve the in vivo detection of luminescent tracers in an interventional setting focus on a number of features. First, the sensitivity of camera systems, meaning the ability to effectively collect luminescence emissions, is a critical feature. In our own studies we noted that in prostate cancer patients, sentinel node detection rates significantly improved from $85.2 \%$ to $93.5 \%$ when the fluorescence imaging system was improved from a standard analog image projection to a more sensitive digital high-definition system (30). It can thus be assumed that further improvement of camera sensitivity is likely to have a comparable positive effect on the usability of fluorescence cameras during interventions. Especially when looking at targeted tracers, where only small tracer amounts reach the target lesion, sensitivity of the camera system will determine whether or not the lesion can be visualized during the intervention. Also, during Cerenkov luminescence imaging of $\beta$ emissions, this effect can be great (1). While dedicated camera development for Cerenkov imaging is still in its infancy, this area of research is already producing some promising results $(31,32)$.

Second, the ability to overlay the fluorescence signal onto the normal white light image may help to further incorporate the use of luminescencebased optical imaging in clinical logistics. In doing so, simultaneous visualization of the fluorescence signal and the anatomic structures (Fig 3, A) enables actual lesion excision under fluorescence guidance (30). While some optical modalities separately detect the white light and fluorescence images before overlaying them, the recent trend is to use a single (multichip) camera to detect both features and provide a fully integrated fluorescence and background image.

Third, while fluorescence emissions such as that of fluorescein can be detected by the naked eye and therefore show up in their natural color (in this case yellow and/or green), red fluorescence gives significantly less contrast over a surgical background and may need some form of artificial coloring for optimal contrast. Near-infrared emissions, which cannot be detected by the 
naked eye, can only be visualized by using a dedicated camera with strict filter settings. Here the near-infrared signal is either presented in black and white or pseudo color coding is used. Figure $3, B$, illustrates the influence that color selection has on the visual interpretation of the image; green gives a much stronger visual contrast with its background than blue and as such can be better detected. Signal intensities can also be presented by using heat-map features, thereby giving more quantitative feedback on, for example, perfusion during surgery (Fig 3, C) (26).

Fourth, the possibility to detect multiple but distinct optical emissions with one camera system (called multispectral imaging or multiplexing) has become a topic of interest. The ability to detect different types of fluorescence signals means that different anatomic, physiologic, and/or molecular parameters can be visualized simultaneously or sequentially. An example of this concept is provided in Figure $3, D$, in which accumulation of the near-infrared tracer $\mathrm{ICG}^{-}{ }^{99 \mathrm{~m}} \mathrm{Tc}$-nanocolloid in the sentinel node (blue, $i$ ) is complemented by detecting the lymphatic flow toward and through the same sentinel node with fluorescein (green/yellow, ii).

Lastly, to make sure the user will not get lost in the level of detail provided, for example when using fiberbased optical devices (Fig 3, E), device placement, tracking, and stabilization are of upmost importance. If fibers are small enough, they can be placed through a cholangioscope (33) or can be passed through a fine needle aspiration cytology needle (34). When placed under radiologic guidance, one may envision that (optical) needle placement may rapidly find an application in minimally invasive treatment strategies. This technology may potentially be of added value during target validation and the assessment of margins prior to the application of minimally invasive treatment strategies such as radiofrequency ablation, microwave ablation, or irreversible electroporation ablation. For the moment, clinical indications of fiber-based technologies are still mostly being defined to luminal organs such as the esophagus and gastrointestinal tract (35).

\section{Luminescence Tracers}

Three main families of luminescencebased exogenous tracers have been reported in clinical trials: fluorescent (Table 1), hybrid (Table 2), and theranostic (Table 3).

\section{Nontargeted Fluorescent Tracers}

Intravenous administration.-Optical means of visualizing the vascular physiology can be used to provide an alternative to contrast-enhanced radiologic techniques. For example, iodinated fluorescein derivatives (55\% iodine by weight) were described as early as 1949 as contrast agents for both $\mathrm{x}$-ray fluoroscopy and fluorescence imaging after intravenous cholecystography (36). In this initial study, gallbladder visualization was achieved in 13 of 35 patients. Since then noniodinated fluorescein, which has a limited toxicity, has received regulatory approval for angiography. The clinical evaluation of fluorescein was followed by the clinical approval of its near-infrared counterpart ICG (37). The vascular retention of ICG, a dye that immediately binds to "large" carrier proteins such as human serum albumin in situ, has been used to assess organ perfusion in different anatomies. Examples include tissue vitality (eg, free-flap reconstruction [38]), partial nephrectomy with selective arterial clamping (39-41), reconstructive surgery of the upper urinary tract (39), or the microscopic assessment of vascular malformations in the brain (37). With regard to the application of ICG for angiography, during partial nephrectomy ICG cannot only be used to locate the artery (Fig 4, A), it can also be used to distinguish normal parenchyma from cysts and tumors; the latter appear hypofluorescent relative to the normal parenchyma (39-41).

Other than perfusion, extravasation of fluorescent tracers into tissue-a feature that is similar to late contrast enhancement at MR imaging-was shown to provide feedback on the physiology. For example, the impaired vascular network of tumors in the eye is routinely assessed by using fluorescein (23), while in a more experimental setting the same has been done for the brain (42). The value of this approach is exemplified by the fact that in two patients (total number of patients in this study was five), in whom $x$-ray angiography failed to show a vascular flush, the finer and sharper contrast obtained by using fluorescence imaging helped to demarcate the tumor tissue based on abnormalities in the vascular bed (42). Alternatively, in 46 patients clinically diagnosed with a brain tumor, fluorescein-based imaging of the needle biopsy specimen and subsequent tumor resection correctly predicted the prescence or absence of a tumor in 44 patients (4). Extravasation of fluorescein also allowed the identification of microscopic changes of lesions located in the gastrointestinal tract (35). The $\mathrm{pH}$ dependancy of fluorescein, where the intensity of the fluorescence emission changes upon changing $\mathrm{pH}$, helped to identify small biologic changes in the lesion. Moreover, morphologic changes and changes in vascularity could be identified by using fluorescein. The latter is illustrated in Figure 3, E, which shows an area with increased permeability and therefore increased fluorescein extravasation (35).

Other dyes that have been shown to accumulate in tumor tissue, for example, breast tumors, are ICG, omocyanine, and methylene blue (43-46). Depending on the dose administrated, omocyanine was able to visualize malignant lesions smaller than $2 \mathrm{~cm}$. However, detection rates strongly decreased with increasing skin-to-lesion distance; $63.6 \%$ of lesions were detected at skin-to-lesion distance of less than $2 \mathrm{~cm}$ whereas only $25 \%$ were detected at a distance greater than $3 \mathrm{~cm}$ (44). Using ICG, Poellinger et al showed a $92.0 \%$ sensitivity and $75.0 \%$ specificity for optical mammography (43). Nevertheless, it was concluded that the technique of optical mammography on its own has insufficient diagnostic accuracy and should be combined with conventional tools (47). Ex vivo fluorescence imaging of breast cancer specimens following intravenously administered methylene blue revealed accumulation of the dye in $83.3 \%$ of the resected tumors (45). 

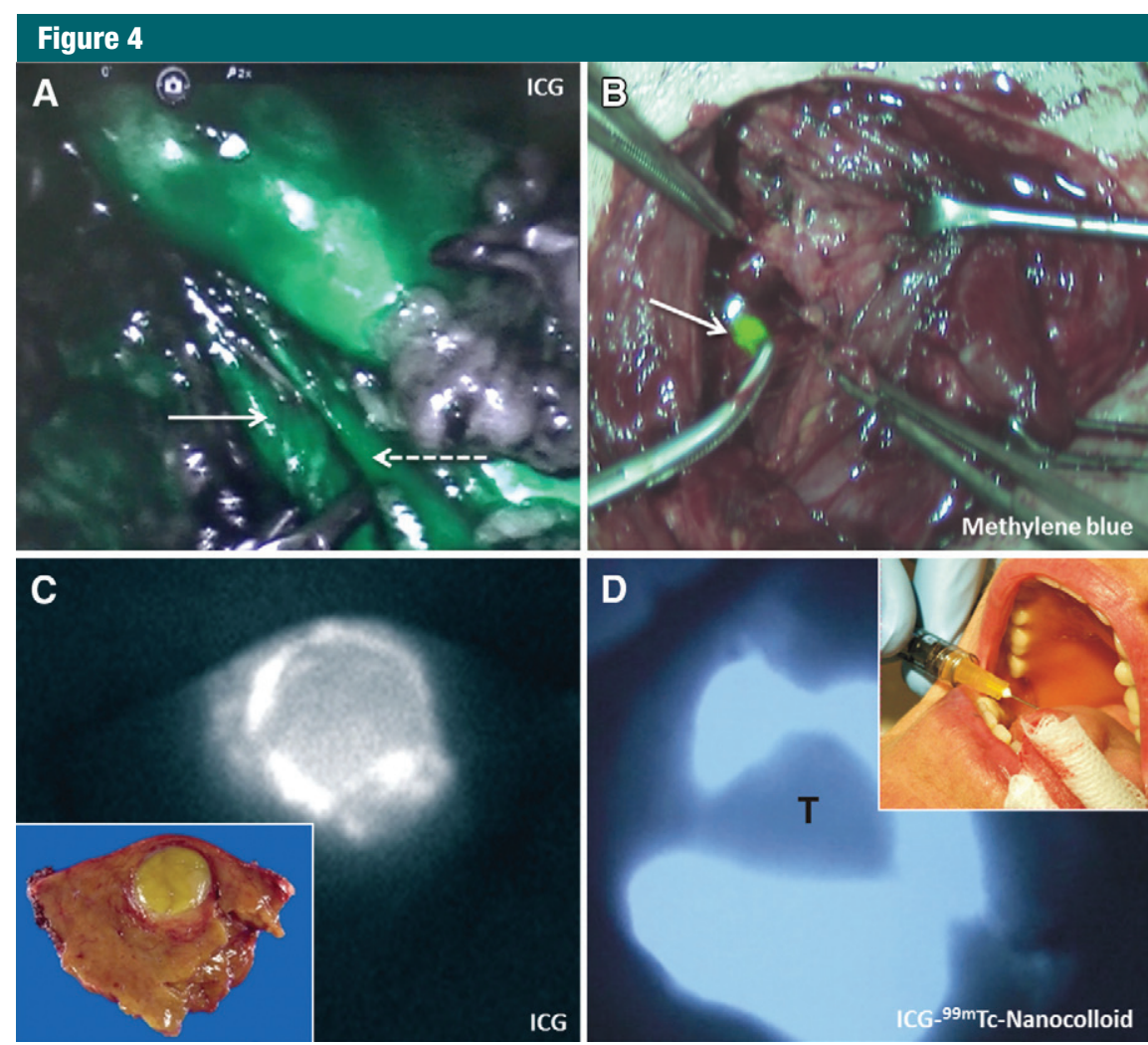

Figure 4: Different applications of nontargeted fluorescence tracers. $A$, Intravenous administration of ICG can be used to visualize blood vessels during partial nephrectomy (fluorescence in green; arrow = clamped artery, dotted arrow = affluent vein; the large green mass is the kidney). $B$, Extravasation of methylene blue can be used to visualize parathyroid adenoma(s) during surgery (fluorescence in green; arrow = location of the parathyroid adenoma). $C$, Disruptions in the hepatic clearance of ICG can be used to visualize hepatocellular lesions in the liver (fluorescence in black and white). The insert shows the corresponding white light image. (Reprinted, with permission, from reference 135.) D, Local injections of ICG-99mTc-nanocolloid surrounding squamous cell carcinoma of the tongue (insert) show prolonged accumulation at the injection site. The fluorescence signal is shown in black and white, with the near-infrared fluorescence signal displayed in white. (Reprinted, with permission, from reference 67.)

Methylene blue has also been routinely used to visualize parathyroid lesions via its true blue color (48). In a similar fashion, recently fluorescence imaging of low-dose methylene blue identified a parathyroid adenoma in nine of 12 patients scheduled for parathyroid surgery; only seven of these nine patients had a positive ${ }^{99 \mathrm{~m} T c-s e s t a m i b i}$ SPECTCT scans (49). Unfortunately, it was not reported what might have caused this differential uptake. Although the uptake mechanisms of these tracers were initially assumed to be identical, the latter finding suggests that there might be a difference in uptake mechanism between the two. Hence, the mode of accumulation of methylene blue is most likely also the result of extravasation.

The pharmacological clearance of nontargeted fluorescent tracers can also be exploited for diagnostic purposes. Functional hepatic clearance of ICG was shown to aid in the visualization of bile ducts during cholangiography $(50,51)$. Impairments in the clearance mechanism, combined with expression of the sodium ion/taurocholate cotransporting peptide and organic anion transporting polypeptide 8 by hepatocellular cancer cells, was shown cause ICG accumulation in the cells, resulting in highly sensitive fluorescence-based identification of these tumors in the liver (52). In this particular study, which included 273 patients, ex vivo fluorescence-based tissue examination enabled the identification of $98.9 \%$ of the hepatocellular carcinoma lesions. Here retention of ICG was similar to what is observed during contrast-enhanced CT or MR imaging of such lesions (53), but the resolution achieved via fluorescence imaging on the surface of the liver was superior. For the detection and/or treatment of deeper-lying lesions, conventional USbased technologies are still mandatory.

Renal clearance of dyes such as fluorescein $(n=12)$ and methylene blue $(n=12)$ was shown to provide a means to visualize ureters $(54,55)$.

Local administration.-Following a local injection, fluorescent dyes can be used for lymphangiography (56-59). Initially radiopaque contrast agents were used, but currently lymphatic mapping is dominated by the use of radiotracers (60-62). Local injections with fluorescein or ICG have been proposed as a more sensitive alternative to blue dye (63) and a more convenient approach than the use of gamma tracing $(64,65)$. Although the lymphatic drainage of ICG and blue dyes is the same, Guo et al (66) reported the fluorescence approach being more sensitive for the detection of the sentinel node, $97.2 \%$ versus $81.3 \%$, respectively. The difference in detection mechanisms, namely the detection of an emitted signal by a light-sensitive camera (fluorescein and/or ICG) versus the detection of light absorbance by the eye (blue dye) provides the most plausible explanation for this difference in sensitivity. The relatively limited tissue penetration of the fluorescence signal, compared with the radioactive signal, can be of value during the detection of lymph nodes located in close proximity to the injection site (67).

Interventional guidance toward nonpalpable lesions detected at preoperative imaging, for example, nonpalpable breast cancer, is often provided via guide-wire localization, radioguided occult lesion localization (using radiocolloids), or radioactive seed localization (using titanium marker seeds filled with a small amount of radioactive iodine). In this application, preoperative 
radiologic imaging such as US or $\mathrm{x}$-ray guidance is used to mark the area that needs further investigation. In a similar fashion, fluorescent markers may be used to stain the area of interest (12). An ICG-labeled radiocolloid (Table 2) has already shown its ability to accumulate at the injection side of oral cavity cancers or prostate for a longer period of time $(67,68)$. In the gastrointestinal tract, a local injection of blue dye is widely used to enable accurate lesion localization during ex vivo (pathologic) examination (69), suggesting the same may be performed in the future by using fluorescent tracers.

Topical administration.-Topical administration of a fluorescent tracer, whereby the tracer is locally sprayed on the area of interest, may be used to provide direct feedback during the intervention. Confocal endomicroscopy of the gastrointestinal mucosa after topical administration of acrilflavine (hydrocholoride) showed increased uptake in areas with an increased cell density (cell nuclei) (70). However, compared with intravenous administration of fluorescein, which allowed labeling of the cells up to the lamina propria, acriflavine was only able to label superficial epithelial cells (71). Cresyl violet, a dye that stains the cytoplasm rather than the nucleus, has been proposed as an alternative for chromoendoscopy and fluorescein endomicroscopy $(n=36)(72)$.

\section{Targeted Fluorescent Tracers}

Intravenous administration. - When targeted fluorescent tracers are used for the evaluation of molecular parameters, the mechanism of action is similar to that of radiotracers: An intravenous bolus administration allows targeting of membrane receptors expressed on the tumor cell surface. In fact, the optical field often explores targeted approaches previously reported by using radiotracers. Since optical labels are relatively large, fluorescent tracers are commonly based on disease-specific peptides or mAbs. Examples of studied molecular targets are CEA $(73,74)$, the folate receptor (75), the receptor tyrosine kinase c-met $(5,76)$, the EGFR $(77)$, and the vascular endothelial growth factor receptor (78).
Unfortunately, in biomarkertargeted interventions that rely on fluorescent tracers only, the limited penetration depth of the fluorescence signal is a considerable weakness. Accumulation in unsuspected areas cannot be assessed, thereby potentially limiting the curative intent of surgery. It is also not possible to use fluorescence to noninvasively stage whether or not the tracer used is of value for an individual patient. An example is the limited success rate of folate FITC reported in patients with ovarian cancer (75). These problems can be solved when a radiotracer for the same target is used to noninvasively assess the targeting ability, location, and spread of the disease.

In contrast, when the location of a lesion is known, real-time and high-resolution superficial identification of lesions become possible. Successful examples of this are the visualization of superficially located ovarian cancer lesions by using folate FITC (in three of 10 patients one or more malignant tumor lesions could be identified intraoperatively; Fig 5, A [75]) and the identification of colorectal neoplasia by using the compound GE137 (Cy5 labeled; Fig 5, B), which targets the c-met receptor (5). For the latter, second-pass fluorescence imaging identified $19 \%$ additional lesions (5).
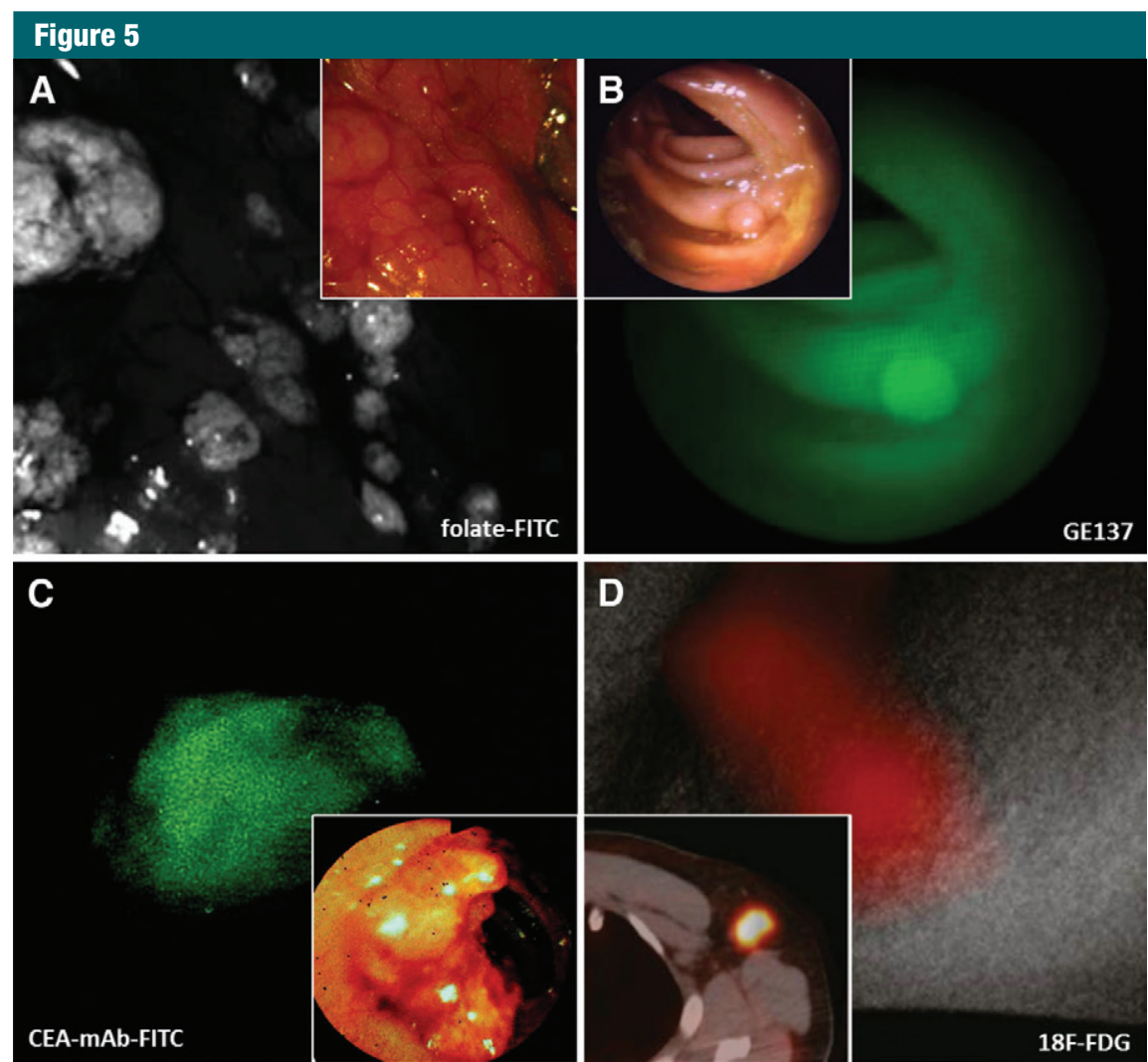

Figure 5: Targeted luminescent tracers. $A$, Folate-FITC can be used to visualize folate receptor-overexpressing lesions of the surface of the ovarium. The fluorescence image is shown in black and white, with the fluorescence signal displayed in white. The insert shows the corresponding white light image. (Reprinted, with permission, from reference 75.) $B$, Intravenous administration of GE137 was shown to highlight cancerous lesions during colonoscopy (fluorescence in green). The insert shows the corresponding white light image. C, Topical administration of CEA-mAb-FITC can enable visualization of cancerous lesions in the lumen of the colon (fluorescence in green). The insert shows the corresponding white light image. (Reprinted, with permission, from reference 74.) $D$, Cerenkov luminescence imaging (luminescence in red) can be used to optically identify ${ }^{18} \mathrm{~F}$ fluorodeoxyglucose accumulation in a lymph node. The insert shows the corresponding PET-CT image. (Reprinted, with permission, from reference 32.) 
Topical administration.-Topical tracer application is increasingly being explored to locally visualize molecular tumor features by using either targeted or activatable fluorescence tracers. This approach makes sense especially when the intention is to further characterize lesions that have been detected previously by radiologic or standard endoscopic methods. Topical methods are therefore not intended to aid in the primary detection of, for example, colorectal neoplasms but to microscopically analyze lesions to predict their histology and/or local extent.

A number of compounds have been evaluated in vivo in humans for the characterization of lesions in the colon, ranging from dysplasia to cancerous lesions. A CEA-targeted mAb (CEA-mAb-FITC; Fig 5, C) was used to target CEA overexpression. In 27 patients, an increased fluorescence signal was found in 19 of 25 carcinomas and three of eight adenomas after local application of the probe (74). A FITC-labeled heptapeptide (VRPMPLQ-FITC) has been used to characterize colorectal neoplasia by binding to a yet unknown molecular target. In 20 patients, confocal endomicroscopy was used to differentiate adenoma from normal tissue after topical adminstration of the dye in the colon, with $81.0 \%$ sensitivity and $82.0 \%$ specificity (79). An anti-EGFR mAB coupled to Alexa Fluor 488 was used to target EGFR-overexpressing colorectal polyps from 37 patients; a specific fluorescence signal and EGFR accumulation could be detected in $18(94.7 \%)$ of 19 carcinomas and 12 $(66.7 \%)$ of 18 adenomas (77).

The FITC-labeled peptide sequence ASYNYDA-GGGSK, isolated with phage display biopanning by using an esophageal cancer cell line, which probably targets cyclophilin A, has been evaluated for the detection of esophageal neoplasia within Barrett esophagus by using confocal endomicroscopy. In 25 patients there were nine true-positive, one false-positive, 34 true-negative, and three false-negative findings for identifying neoplasia, resulting in a positive predictive value of $90.0 \%$ and a negative predictive value of $91.9 \%(80)$.
Topical administration of enzymeactivated tracers is attractive in that they should be able to achieve higher target-to-background ratios with no background signal from the quenched, inactivated tracer and a multiplication of the signal due to enzymatic activation of the probe at the site of enzyme overexpression. In ex vivo human breast and lung cancer tissues it was shown that the enzyme $\gamma$-glutamyltranspeptidase was able to activate the topical dye gGlu-HMRG, resulting in fluorescent lesions (81). The future challenge mainly lies in the identification of identical, or complementary, radiologic features that can be used to guide physicians in their topical administration of (activatable) fluorescent tracers. In essence, the guidance required for topical tracer administration is highly similar to the guidance needed to acquire biopsy specimens of a diseased area.

\section{Hybrid Tracers}

Tracers combining a radioactive and a luminescent signature allow for initial identification of the lesion at conventional preinterventional imaging (eg, SPECT or PET), followed by intraoperative luminescence guidance. Here the radioactive signal of hybrid tracers overcomes the major shortcoming of optical imaging, namely in-depth signal penetration. On top, it opens the field for the use of non-near-infrared luminescence emissions during interventional guidance; with the radioactive signal providing an in-depth view, optical detection can be of value even when it is highly superficial in nature.

Two subclasses of luminescencebased hybrid tracers have been reported for clinical use, namely tracers that combine a radio and fluorescent label and radioisotopes that enable Cerenkov luminescence imaging (Table 2). Other than the fluorescent tracers described above, hybrid tracers have only been used in combination with intravenous and local administration.

Combination of a radioisotope and a fluorescent dye.-To date, only two combined radioactive and fluorescent hybrid approaches that have been reported in clinical studies use
${ }^{131} \mathrm{I}$-fluorescein $(82,83)$ and $\mathrm{ICG}^{-99 \mathrm{~m}} \mathrm{Tc}$ nanocolloid $(10,30,84,85)$. For the excitation and emission of these agents, the same rules apply as for the conventional fluorescent tracers (illustrated in Fig 2).

Intravenous administration of ${ }^{131} \mathrm{I}-$ fluorescein was first reported in 1948 as a vascular contrast agent for the detection of disrupted vascularization of brain tumors (82). Here, the iodine compound was used to noninvasively assess the degree of accumulation prior to the intervention. Preoperative imaging findings overlapped with surgical findings in 11 of 12 patients (82).

The hybrid tracer ICG- $^{-99 m}$ Tc-nanocolloid is a lymphatic agent that is composed of the commercially available radiolabeled human serum albumin colloid (nanocolloid) and ICG (84). Similar to the parental radiocolloid, following a local peritumoral injection the hybrid tracer accumulates in sentinel nodes (84). This compound has been evaluated in more than 500 patients and has proven valuable for sentinel node procedures at different sites, such as melanoma, penile cancer, and prostate cancer $(10,30,84,85)$. The combined approach was shown to be superior to radioguidance technologies alone. It has aided in optimizing the logistics and improved the detection of sentinel nodes residing in a complex anatomy (Movie [online]).

Although the fluorescent properties of methylene blue have not yet been explored for radioiodinated $\left({ }^{123} \mathrm{I},{ }^{125} \mathrm{I}\right.$, or $\left.{ }^{131} \mathrm{I}\right)$ methylene blue, in theory this compound can be considered a hybrid tracer. Practically this would mean that all the diagnostic applications of methylene blue can be complemented by indepth in vivo guidance via gamma signal detection. Early studies indicated methylene blue an unsatisfactory parathyroid imaging agent (86), but is said to be of value in the detection of melanoma metastases (87). In melanoma, gamma tracing of radioiodinated methylene blue confirmed the existence of approximately $80 \%$ of the lesions and enabled detection of secondary lesions in six of 12 patients. Alternatively, the radiolabel on methylene blue may be 
used to provide radioguidance during lymphatic mapping, as was shown in an initial feasibility study in 12 women scheduled for breast cancer-based sentinel node biopsy (88).

While the use of radiolabeled fluorescein and methylene blue indicates that iodination may be acceptable for small molecules, the success of ICG99m Tc-nanocolloid may also be representative for fluorescence labeling of large protein-based radiotracers such as antibodies. Theoretically, radiotracers used for SPECT, PET, and radioguided surgery can all be chemically modified into hybrid tracers. The same goes for US and MR imaging contrast agents. However, one should consider that modification of an established radiotracer may negatively influence its affinity, pharmacokinetics, and biodistribution $(89,90)$.

Use of a radioisotope that also allows for Cerenkov luminescence.-Despite having an emission peak in a part of the light spectrum that has minimal tissue penetration $(<200 \mathrm{~nm})$, the first studies in humans yielded promising results. A therapeutic dose of ${ }^{131} \mathrm{I}$ (550 $\mathrm{MBq}$ ) was used for luminescence imaging of the thyroid in a single patient (31). In patients with lymphoma $(n=2)$, lung $(n=1)$, or breast $(n=$ 1) cancer, after routine ${ }^{18} \mathrm{~F}$-FDG PETCT imaging (450 MBq), Cerenkov luminescence imaging revealed the site of accumulation in the ${ }^{18} \mathrm{~F}$-FDG PETpositive lymph nodes in the neck and axilla (Fig 5, D) (32). Although imaging cannot yet occur at video rate, the current detection sensitivity achieved is exceptional when one considers that the photon flux from ${ }^{18} \mathrm{~F}$ is orders of magnitudes smaller than that of widely used fluorescent dyes (1). Crucial in the success of this imaging technique is the ability to determine the area of interest prior to the intervention by using the acquired PET-CT images.

\section{Theranostic Tracers}

Next to their use for therapy purposes, theranostic compounds can also provide optical feedback during interventions. For the excitation and emission of these agents, similar rules apply
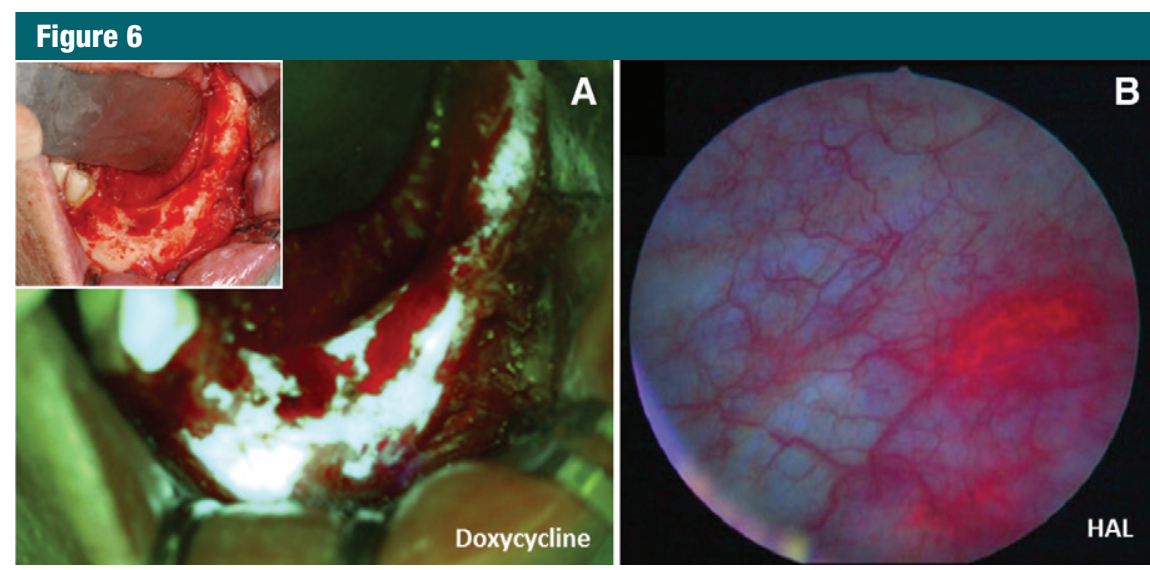

Figure 6: Therapeutic agents that can be used for luminescence imaging. $A$, After administration of doxycycline (fluorescence in yellow), necrotic areas in the jaw become hypofluorescent. The insert shows the corresponding white light image. (Reprinted, with permission, from reference 95.) $B$, Local instillation of hexyl aminolevulinate (HAL) (fluorescence in red) can be used to identify cancerous lesions in the bladder wall. (Reprinted, with permission, from reference 136.)

as those for fluorescent and hybrid tracers (Fig 2). An example of a group of theranostic agents can be found in cycline derivatives. Following internalization, fluorescence imaging of tetracycline, doxycycline, and demecocycline was shown to distinguish viable bone tissue from the nonfluorescent necrotic tissue (91-96). With doxocycline, prior to fluorescence imaging patients required the drug to be administered in a 10-day administration regimen (Fig 6, A) (95).

Alternatively, photodynamic therapy, a minimally invasive ablation technology, can be combined with photodynamic diagnosis (PDD). This procedure is based on the concept of intravenous administration of a compound that accumulates more specifically in tumor cells than in normal tissue (extensively reviewed by Celli et al [97]). Following entrapment of the agent in malignant, inflammatory, and/or infected tissue (98), it as metabolized into the fluorescent compound protoporphyrin IX; well-known protoporphyrin IX precursors are ALA, hexyl ester of ALA (HAL), and methyl ester of ALA (MAL) (Table 3). Although 5-ALA, HAL, and MAL all have all been used in similar applications such as PDD of brain tumors, skin, and bladder cancer, HAL and MAL are considered superior to 5-ALA (97). This effect may well be related to the formulation used, since the ability of these compounds to induce more protoporphyrin IX is still somewhat controversial (99). Following intravenous administration of 5-ALA, fluorescence-guided malignant glioma excision versus white-light-based excision was evaluated in a randomized cohort of 322 patients and yielded a higher 6-month progression-free survival than that in the patients assigned to white light only $(41.0 \%$ vs $21.1 \%$, respectively) (100). Alternatively, bladder-instilled HAL (Fig 6,B) improved the accuracy of white-light cystoscopy in lesion detectability, from $75.3 \%$ to $86.7 \%$, respectively (101). The preformed porphyrin derivative metatetrahydroxyphenylchlorin has been used for PDD of brain and oral cavity tumors. In 22 patients, a sensitivity of $87.9 \%$ and a specificity of $95.0 \%$ were found for brain tumor detection (102).

Hypericin, a natural compound derived from the Hypericum perforatum plant (mechanism of tumor accumulation unknown), has been successfully used for the detection of bladder cancer, with a specificity ranging 91\%-98\%. Compared with other PDD agents, it was claimed to reduce the incidence of false-negative findings (103).

A clear downside of the abovementioned theranostic agents is the 
unavailability of a complementary noninvasive imaging method that can be used to select patients who would benefit most from the above procedures.

\section{Gurrent Status and Future Directions}

Luminescence-based optical imaging has already been applied in a variety of interventional settings. From these studies the strengths and weaknesses of the technology can be derived. The main strength of luminescence imaging lies in its ability to provide real-time feedback regarding the location and margins of a lesion on a microscopic or molecular level. The main weakness of luminescence imaging is its intrinsic inability to look deep into the tissue. On its own, it can, therefore, only be of value for the identification of lesions with a known or superficial location in a lumen or cavity accessible with a camera.

For luminescence guidance to be minimally invasive, patients should have already been accurately staged by using noninvasive radiologic or nuclear medicine imaging technologies. This is especially important when an attempt is made to depict molecular features of a disease. When a preinterventional imaging approach matches the interventional use of luminescence imaging, potentially a "best of both worlds" scenario is generated, with detailed feedback on the disease and its extent. Hence, luminescence imaging is unlikely to replace existing radiologic and nuclear medicine technologies, but will provide a valuable add-on to existing interventional imaging approaches.

The efficient integration of luminescence imaging with other radiologic- or nuclear-medicine-based modalities may be accomplished by using either sequential imaging with different tracers or by using a hybrid imaging approach, wherein a single tracer can be detected with two complementary modalities. If the location of a lesion can already be defined accurately prior to the intervention, a luminescent tracer can be used to visualize complementary rather than identical features, for example, bloodflow kinetics (26). The reverse is also true, as radiologic modalities such as
C-arm, CT, or US may be used to provide anatomic context for physiologic or molecular features depicted with luminescence imaging technologies.

\section{Integration of Luminescence-based Imaging in the Clinical Workflow}

The availability of this "new" type of imaging modality presents practical challenges when it comes to its integration into the clinical workflow. For that reason, future developments should also improve the clinical utility of the technique. For example, in open surgery, most of the currently available luminescence imaging camera systems require the lights in the operating room to be dimmed or switched off completely to allow for the optimal identification of (near-infrared) luminescence signals $(5,84,104)$. Despite the ongoing developments to integrate luminescence imaging into surgical goggles $(21,22)$, the feedback provided by luminescence imaging is generally depicted on a screen. In open-surgery procedures, this feature complicates the routine and requires training before it can be used optimally. This is not a limiting factor in microscopic or endoscopic applications, where interventions are already routinely performed by using an on-screen video feed, and integration of luminescence-based information only has a limited influence on the workflow $(5,13,26)$. One unique example where luminescence imaging has even become an integral part of the surgical tools is found in robotic surgery (39-41); the da Vinci Si robot (Intuitive Surgical) can be equipped with an endoscope suitable for near-infrared imaging.

\section{Optical Properties of Clinically Used Fluorescent Dyes}

For interventional guidance, there has been a scientific push toward the use of near-infrared dyes. Unfortunately, the signal intensity of near-infrared dyes is severely limited by their low photon flux. The ideal exogenous fluorescent dye combines a high absorption coefficient with a high quantum yield, yielding optimal conversion of light. The limited emission strength of near-infrared dyes is partially compensated by the fact that excitation and emission of these dyes occur in the "most ideal" section of the light spectrum, yielding minimal autofluorescence and signal attenuation. Nevertheless, with the exception of ICG, CW800, and omocyanine, all other luminescent tracers that have been evaluated in clinical trials emit outside the near-infrared spectrum (200-780 nm; Tables 1-3). Note that the term near-infrared is often wrongly used to describe excitations and emissions below $780 \mathrm{~nm}$.

For molecular luminescence imaging in particular, next to the near-infrared tracers, Cerenkov luminescence imaging, PDD contrast agents, fluorescein derivatives, and a Cy5 derivative have shown their value in the clinic. This suggests that the tissue penetration efficiency of the luminescence signal is not necessarily a limiting factor for their clinical use. Depending on the application, the best balance between signal intensity and penetration depth should be sought.

\section{Future Targets for Luminescence Imaging}

Tables 1-3 indicate that luminescence imaging can be used to depict a variety of anatomic, physiologic, and molecular features. Clinical trials have boosted preclinical efforts to improve the luminescence properties and explore new targets, yielding a wide variety of potential imaging agents. From a chemica perspective, there are few restrictions for the future design of different luminescent dyes and new tracers.

Hybrid approaches are increasingly being pursued in preclinical studies. The chemical variations are only limited by the number of radioisotopes available for imaging purposes. While essentially any luminescence tracer can be chemically modified into a hybrid derivative $(90,105)$, it is not trivial to do the reverse; the size of a fluorescent dye may be noticeably larger than the size of the radioisotope used. Although the combination of luminescence and nuclear labels provides the best fit with regard to interventional use and contrast sensitivity, combinations can also be made between luminescence and, for example, MR imaging contrast (106). 


\section{Clinical Availability}

While compounds such as ICG and fluorescein have found their way into clinical routine, most of the other tracers discussed have not yet progressed beyond small first human trials. Even in more routine applications, such as sentinel node procedures, clinically approved compounds are often still being used offlabel. On the basis of the amount of successful trials reported, it is highly likely that in the near future the amount of luminescent tracers will expand. However, all of these new luminescent tracers will need to be approved for human use individually. Combined with the costs associated with getting this approval, this may limit the number of tracers that will find their way into the clinical routine. In this setting, Cerenkov luminescence imaging, an imaging option that is directly derived from the already approved PET tracers, seems to have a big advantage.

\section{Conclusion}

Although much has to be done before the highly superficial luminescencebased imaging technologies replace existing guidance technologies, the outcome of a variety of clinical trials with luminescent tracers suggests the technology may help provide physicians with real-time and detailed (molecular) feedback during an intervention. More extensive multicenter trials are, however, required to determine how the use of luminescence technologies will affect clinical outcome.

Disclosures of Conflicts of Interest: F.W.B.v.L. Activities related to the present article: received grants from Dutch cancer society. Activities not related to the present article: disclosed no relevant relationships. Other relationships: disclosed no relevant relationships. J.C.H.H. Activities related to the present article: institution received money from GE Healthcare; subcontracted by the Centre for Human Drug Research, Leiden, to perform endoscopies for human safety studies and a patient study of GE-137 optical molecular imaging agent (Euro 50,000). Activities not related to the present article: disclosed no relevant relationships. Other relationships: disclosed no relevant relationships. A.R.v.E. disclosed no relevant relationships.

The authors declare to have no conflicts of interest and no commercial links with the industry. They, however, are or have been involved in clinical fluorescence-guided surgery trials that KARL
STORZ GmbH \& Co. KG, Eurorad, Hamamatsu Photonics, and GE Healthcare support(ed) by making luminescence imaging hardware available.

\section{References}

1. Chin PT, Welling MM, Meskers SC, Valdes Olmos RA, Tanke H, van Leeuwen FW. Optical imaging as an expansion of nuclear medicine: Cerenkov-based luminescence vs fluorescence-based luminescence. Eur J Nucl Med Mol Imaging 2013;40(8):1283-1291.

2. Yang $X$. Interventional molecular imaging. Radiology 2010;254(3):651-654.

3. Policard A. Étude sur les aspects offerts par des tumeurs expérimentales examinées à la lumière de Wood. C R Soc Biol Paris 1924;91:1423-1424

4. Moore GE, Peyton WT, et al. The clinical use of fluorescein in neurosurgery: the localization of brain tumors. J Neurosurg 1948;5(4): 392-398.

5. Burggraaf J, Kamerlin IM, Gordon PB, et al. Detection of colorectal neoplasia in vivo in humans using an intravenously administered fluorescent peptide targeted against c-Met and fluorescence colonoscopy: a proof of concept study. Nat Med (in press).

6. Jaffray DA. Image-guided radiotherapy: from current concept to future perspectives. Nat Rev Clin Oncol 2012;9(12):688-699.

7. Mariani G, Bruselli L, Kuwert T, et al. A review on the clinical uses of SPECT/CT. Eur J Nucl Med Mol Imaging 2010;37(10):19591985.

8. Navab N, Blum T, Wang LJ, Okur A, Wendler T. First deployments of augmented reality in operating rooms. Computer 2012; 45(7):48-55.

9. van den Berg NS, Valdés-Olmos RA, van der Poel HG, van Leeuwen FW. Sentinel lymph node biopsy for prostate cancer: a hybrid approach. J Nucl Med 2013; 54(4):493-496.

10. Brouwer OR, van den Berg NS, Mathéron $\mathrm{HM}$, et al. A hybrid radioactive and fluorescent tracer for sentinel node biopsy in penile carcinoma as a potential replacement for blue dye. Eur Urol 2014;65(3):600-609.

11. Sheppard N, Willis HA, Rigg JC. Names, symbols, definitions and units of quantities in optical spectroscopy. Pure Appl Chem 1985;57(1):105-120.

12. Chin PT, Beekman CA, Buckle T, Josephson L, van Leeuwen FW. Multispectral visualization of surgical safety-margins using fluorescent marker seeds. Am J Nucl Med Mol Imaging 2012;2(2):151-162.

13. van der Poel HG, Buckle T, Brouwer OR, Valdés Olmos RA, van Leeuwen FW. In- traoperative laparoscopic fluorescence guidance to the sentinel lymph node in prostate cancer patients: clinical proof of concept of an integrated functional imaging approach using a multimodal tracer. Eur Urol 2011;60(4):826-833.

14. Garcia-Allende PB, Glatz J, Koch M, Ntziachristos V. Enriching the interventional vision of cancer with fluorescence and optoacoustic imaging. $\mathrm{J}$ Nucl Med 2013;54(5):664-667.

15. Meikle SR, Kench P, Kassiou M, Banati RB. Small animal SPECT and its place in the matrix of molecular imaging technologies. Phys Med Biol 2005;50(22):R45-R61.

16. Bunschoten A, Buckle T, Kuil J, et al. Targeted non-covalent self-assembled nanoparticles based on human serum albumin. Biomaterials 2012;33(3):867-875.

17. Vahrmeijer AL, Frangioni JV. Seeing the invisible during surgery. $\mathrm{Br} \mathrm{J}$ Surg 2011;98(6):749-750.

18. Troyan SL, Kianzad V, Gibbs-Strauss SL, et al. The FLARE intraoperative near-infrared fluorescence imaging system: a first-inhuman clinical trial in breast cancer sentinel lymph node mapping. Ann Surg Oncol 2009;16(10):2943-2952.

19. Hirche C, Engel H, Kolios L, et al. An experimental study to evaluate the Fluobeam 800 imaging system for fluorescence-guided lymphatic imaging and sentinel node biopsy. Surg Innov 2013;20(5):516-523.

20. Themelis G, Yoo JS, Soh KS, Schulz R, Ntziachristos V. Real-time intraoperative fluorescence imaging system using lightabsorption correction. J Biomed Opt 2009; 14(6):064012.

21. Liu Y, Akers WJ, Bauer AQ, et al. Intraoperative detection of liver tumors aided by a fluorescence goggle system and multimodal imaging. Analyst (Lond) 2013;138(8):2254-2257.

22. Liu Y, Bauer AQ, Akers WJ, et al. Handsfree, wireless goggles for near-infrared fluorescence and real-time image-guided surgery. Surgery 2011;149(5):689-698.

23. Rodrigues EB, Costa EF, Penha FM, et al. The use of vital dyes in ocular surgery. Surv Ophthalmol 2009;54(5):576-617.

24. Babu R, Adamson DC. Fluorescence-guided malignant glioma resections. Curr Drug Discov Technol 2012;9(4):256-267.

25. Sanai N. Emerging operative strategies in neurosurgical oncology. Curr Opin Neurol 2012;25(6):756-766.

26. Chen SF, Kato Y, Oda J, et al. The application of intraoperative near-infrared indocyanine green videoangiography and analysis 
of fluorescence intensity in cerebrovascular surgery. Surg Neurol Int 2011;2:42.

27. Goetz M, Malek NP, Kiesslich R. Microscopic imaging in endoscopy: endomicroscopy and endocytoscopy. Nat Rev Gastroenterol Hepatol 2014;11(1):11-18.

28. Zehri AH, Ramey W, Georges JF, et al. Neurosurgical confocal endomicroscopy: A review of contrast agents, confocal systems, and future imaging modalities. Surg Neurol Int 2014;5:60.

29. Goetz M, Deris I, Vieth M, et al. Near-infrared confocal imaging during mini-laparoscopy: a novel rigid endomicroscope with increased imaging plane depth. J Hepatol 2010;53(1):84-90.

30. KleinJan GH, van den Berg NS, Brouwer OR, et al. Optimisation of fluorescence guidance during robot-assisted laparoscopic sentinel node biopsy for prostate cancer. Eur Urol 2014;66(6):991-998.

31. Spinelli AE, Ferdeghini M, Cavedon C, et al. First human Cerenkography. J Biomed Opt 2013;18(2):20502.

32. Thorek DL, Riedl CC, Grimm J. Clinical Cerenkov luminescence imaging of (18)FFDG. J Nucl Med 2014;55(1):95-98.

33. Meining A, Chen YK, Pleskow D, et al. Direct visualization of indeterminate pancreaticobiliary strictures with probe-based confocal laser endomicroscopy: a multicenter experience. Gastrointest Endosc 2011;74(5):961-968.

34. Konda VJ, Meining A, Jamil LH, et al. A pilot study of in vivo identification of pancreatic cystic neoplasms with needlebased confocal laser endomicroscopy under endosonographic guidance. Endoscopy 2013;45(12): 1006-1013.

35. Goetz M. Confocal laser endomicroscopy: applications in clinical and translational science-a comprehensive review. ISRN Pathol 2012;2012:387145

36. Smith MJ, Moore GE. Sodium tetraiodophthalicfluorescein for intravenous cholecystography. Radiology 1949;53(4):552-554.

37. Alander JT, Kaartinen I, Laakso A, et al. A review of indocyanine green fluorescent imaging in surgery. Int $\mathrm{J}$ Biomed Imaging 2012;2012:940585.

38. Holm C, Dornseifer U, Sturtz G, Ninkovic M. Sensitivity and specificity of ICG angiography in free flap reexploration. J Reconstr Microsurg 2010;26(5):311-316.

39. Bjurlin MA, Gan M, McClintock TR, et al. Near-infrared fluorescence imaging: emerging applications in robotic upper urinary tract surgery. Eur Urol 2014;65(4):793-801.
40. Krane LS, Manny TB, Hemal AK. Is near infrared fluorescence imaging using indocyanine green dye useful in robotic partial nephrectomy: a prospective comparative study of 94 patients. Urology 2012; 80(1):110-116.

41. Tobis S, Knopf JK, Silvers CR, et al. Near infrared fluorescence imaging after intravenous indocyanine green: initial clinical experience with open partial nephrectomy for renal cortical tumors. Urology 2012; 79(4):958-964.

42. Feindel W, Yamamoto YL, Hodge CP. Intracarotid fluorescein angiography: a new method for examination of the epicerebral circulation in man. Can Med Assoc J 1967;96(1):1-7.

43. Poellinger A, Burock S, Grosenick D, et al. Breast cancer: early- and late-fluorescence near-infrared imaging with indocyanine green-a preliminary study. Radiology 2011; 258(2):409-416.

44. Poellinger A, Persigehl T, Mahler M, et al. Near-infrared imaging of the breast using omocianine as a fluorescent dye: results of a placebo-controlled, clinical, multicenter trial. Invest Radiol 2011;46(11):697-704.

45. Tummers QR, Verbeek FP, Schaafsma BE, et al. Real-time intraoperative detection of breast cancer using near-infrared fluorescence imaging and Methylene Blue. Eur J Surg Oncol 2014;40(7):850-858.

46. van de Ven S, Wiethoff A, Nielsen T, et al. A novel fluorescent imaging agent for diffuse optical tomography of the breast: first clinical experience in patients. Mol Imaging Biol 2010;12(3):343-348.

47. Poellinger A. Near-infrared imaging of breast cancer using optical contrast agents. J Biophotonics 2012;5(11-12):815-826.

48. Patel HP, Chadwick DR, Harrison BJ, Balasubramanian SP. Systematic review of intravenous methylene blue in parathyroid surgery. Br J Surg 2012;99(10):1345-1351.

49. van der Vorst JR, Schaafsma BE, Verbeek FP, et al. Intraoperative near-infrared fluorescence imaging of parathyroid adenomas with use of low-dose methylene blue. Head Neck 2014;36(6):853-858.

50. Ishizawa $\mathrm{T}$, Tamura $\mathrm{S}$, Masuda $\mathrm{K}$, et al. Intraoperative fluorescent cholangiography using indocyanine green: a biliary road map for safe surgery. J Am Coll Surg 2009; 208(1):e1-e4.

51. Sugimoto M. Recent advances in visualization, imaging, and navigation in hepatobiliary and pancreatic sciences. J Hepatobiliary Pancreat Sci 2010;17(5):574-576.

52. Ishizawa $\mathrm{T}$, Masuda $\mathrm{K}$, Urano $\mathrm{Y}$, et al. Mechanistic background and clinical appli- cations of indocyanine green fluorescence imaging of hepatocellular carcinoma. Ann Surg Oncol 2014;21(2):440-448.

53. Muhi A, Ichikawa $\mathrm{T}$, Motosugi $\mathrm{U}$, et al. Diagnosis of colorectal hepatic metastases: comparison of contrast-enhanced CT, contrast-enhanced US, superparamagnetic iron oxide-enhanced MRI, and gadoxetic acid-enhanced MRI. J Magn Reson Imaging 2011;34(2):326-335.

54. Udshmadshuridze NS, Asikuri TO. Intra-operative imaging of the ureter with sodium fluorescein [in German]. Z Urol Nephrol 1988;81(10):635-639.

55. Verbeek FP, van der Vorst JR, Schaafsma $\mathrm{BE}$, et al. Intraoperative near infrared fluorescence guided identification of the ureters using low dose methylene blue: a first in human experience. J Urol 2013;190(2):574-579.

56. Cooper SG, Maitem AN, Richman AH. Fluorescein labeling of lymphatic vessels for lymphangiography. Radiology 1988; 167(2):559-560.

57. Isenring G, Franzeck UK, Bollinger A. Fluorescence microlymphography of the medial malleolus in healthy humans and in patients with primary lymphedema [in German]. Schweiz Med Wochenschr 1982;112(7): 225-231.

58. Kitai T, Inomoto T, Miwa M, Shikayama T. Fluorescence navigation with indocyanine green for detecting sentinel lymph nodes in breast cancer. Breast Cancer 2005;12(3):211-215.

59. Rasmussen JC, Tan IC, Marshall MV, Fife CE, Sevick-Muraca EM. Lymphatic imaging in humans with near-infrared fluorescence. Curr Opin Biotechnol 2009;20(1):74-82.

60. Mariani G, Erba P, Manca G, et al. Radioguided sentinel lymph node biopsy in patients with malignant cutaneous melanoma: the nuclear medicine contribution. J Surg Oncol 2004;85(3):141-151.

61. Mariani G, Erba P, Villa G, et al. Lymphoscintigraphic and intraoperative detection of the sentinel lymph node in breast cancer patients: the nuclear medicine perspective. J Surg Oncol 2004;85(3):112-122.

62. Van Den Berg NS, Buckle T, Kleinjan GI, et al. Hybrid tracers for sentinel node biopsy. Q J Nucl Med Mol Imaging 2014;58(2):193-206.

63. Jung SY, Kim SK, Kim SW, et al. Comparison of sentinel lymph node biopsy guided by the multimodal method of indocyanine green fluorescence, radioisotope, and blue dye versus the radioisotope method in breast cancer: a randomized controlled trial. Ann Surg Oncol 2014;21(4):1254-1259. 
64. Povoski SP, Neff RL, Mojzisik CM, et al. A comprehensive overview of radioguided surgery using gamma detection probe technology. World J Surg Oncol 2009;7:11.

65. Rambaldi PF, Cuccurullo V, Briganti V, Mansi L. The present and future role of (111)In pentetreotide in the PET era. Q $\mathrm{J}$ Nucl Med Mol Imaging 2005;49(3):225-235.

66. Guo W, Zhang L, Ji J, Gao W, Liu J, Tong M. Breast cancer sentinel lymph node mapping using near-infrared guided indocyanine green in comparison with blue dye. Tumour Biol 2014;35(4):3073-3078.

67. van den Berg NS, Brouwer OR, Klop WM, et al. Concomitant radio- and fluorescenceguided sentinel lymph node biopsy in squamous cell carcinoma of the oral cavity using ICG-(99m)Tc-nanocolloid. Eur J Nucl Med Mol Imaging 2012;39(7):1128-1136.

68. Buckle T, Brouwer OR, Valdés Olmos RA, van der Poel HG, van Leeuwen FW. Relationship between intraprostatic tracer deposits and sentinel lymph node mapping in prostate cancer patients. J Nucl Med 2012;53(7):1026-1033.

69. Yeung JM, Maxwell-Armstrong C, Acheson AG. Colonic tattooing in laparoscopic surgery - making the mark? Colorectal Dis 2009;11(5):527-530.

70. Polglase AL, McLaren WJ, Skinner SA, Kiesslich R, Neurath MF, Delaney PM. A fluorescence confocal endomicroscope for in vivo microscopy of the upper- and the lower-GI tract. Gastrointest Endosc 2005;62(5):686-695.

71. Kiesslich R, Burg J, Vieth M, et al. Confocal laser endoscopy for diagnosing intraepithelial neoplasias and colorectal cancer in vivo. Gastroenterology 2004;127(3):706-713.

72. Goetz M, Toermer T, Vieth M, et al. Simultaneous confocal laser endomicroscopy and chromoendoscopy with topical cresyl violet. Gastrointest Endosc 2009;70(5):959-968.

73. Goldenberg DM. Cancer imaging with CEA antibodies: historical and current perspectives. Int J Biol Markers 1992;7(3):183-188.

74. Keller R, Winde G, Terpe HJ, Foerster EC, Domschke W. Fluorescence endoscopy using a fluorescein-labeled monoclonal antibody against carcinoembryonic antigen in patients with colorectal carcinoma and adenoma. Endoscopy 2002;34(10):801-807.

75. van Dam GM, Themelis G, Crane LM, et al. Intraoperative tumor-specific fluorescence imaging in ovarian cancer by folate receptor- $\alpha$ targeting: first in-human results. Nat Med 2011;17(10):1315-1319.

76. Kim EM, Park EH, Cheong SJ, et al. Characterization, biodistribution and small-ani- mal SPECT of I-125-labeled c-Met binding peptide in mice bearing c-Met receptor tyrosine kinase-positive tumor xenografts. Nucl Med Biol 2009;36(4):371-378.

77. Liu J, Zuo X, Li C, et al. In vivo molecular imaging of epidermal growth factor receptor in patients with colorectal neoplasia using confocal laser endomicroscopy. Cancer Lett 2013;330(2):200-207.

78. Gaykema SB, Brouwers AH, Lub-de Hooge $\mathrm{MN}$, et al. 89Zr-bevacizumab PET imaging in primary breast cancer. J Nucl Med 2013;54(7):1014-1018.

79. Hsiung PL, Hardy J, Friedland S, et al. Detection of colonic dysplasia in vivo using a targeted heptapeptide and confocal microendoscopy. Nat Med 2008;14(4):454-458.

80. Sturm MB, Joshi BP, Lu S, et al. Targeted imaging of esophageal neoplasia with a fluorescently labeled peptide: first-in-human results. Sci Transl Med 2013;5(184):84ra61.

81. Urano Y, Kamiya M, Hino H, et al. Rapid and sensitive imaging of cancer cells in resected human breast and lung tissues by novel fluorescence probes for GGT [abstr]. Mol Imaging Biol 2013;15(Suppl A):2-1590.

82. Moore GE. Use of radioactive diiodofluorescein in the diagnosis and localization of brain tumors. Science 1948;107(2787): 569-571.

83. Moore GE, Peyton WT, et al. The clinical use of sodium fluorescein and radioactive diiodofluorescein in the localization of tumors of the central nervous system. Minn Med 1948;31(10):1073-1076.

84. Brouwer OR, Buckle T, Vermeeren L, et al. Comparing the hybrid fluorescentradioactive tracer indocyanine green$99 \mathrm{mTc}$-nanocolloid with $99 \mathrm{mTc}$-nanocolloid for sentinel node identification: a validation study using lymphoscintigraphy and SPECT/CT. J Nucl Med 2012;53(7): 1034-1040.

85. van den Berg NS, Brouwer OR, Schaafsma $\mathrm{BE}$, et al. Multimodal surgical guidance during sentinel node biopsy for melanoma: combined gamma tracing and fluorescence imaging of the sentinel node through use of the hybrid tracer indocyanine green99mTc-nanocolloid. Radiology 2014 Dec 17. [Epub ahead of print]

86. Blower PJ, Kettle AG, O’Doherty MJ, Collins RE, Coakley AJ. 123I-methylene blue: an unsatisfactory parathyroid imaging agent. Nucl Med Commun 1992;13(7):522-527.

87. Link EM, Blower PJ, Costa DC, et al. Early detection of melanoma metastases with radioiodinated methylene blue. Eur J Nucl Med 1998;25(9):1322-1329.
88. Cundiff JD, Wang YZ, Espenan G, et al. A phase I/II trial of $125 \mathrm{I}$ methylene blue for one-stage sentinel lymph node biopsy. Ann Surg 2007;245(2):290-296.

89. Kuil J, Buckle T, Oldenburg J, et al. Hybrid peptide dendrimers for imaging of chemokine receptor 4 (CXCR4) expression. Mol Pharm 2011;8(6):2444-2453.

90. Kuil J, Buckle T, Yuan H, et al. Synthesis and evaluation of a bimodal CXCR4 antagonistic peptide. Bioconjug Chem 2011; 22(5):859-864.

91. Dahners LE, Bos GD. Fluorescent tetracycline labeling as an aid to debridement of necrotic bone in the treatment of chronic osteomyelitis. J Orthop Trauma 2002;16(5):345-346.

92. Frost HM. Tetracycline-based histological analysis of bone remodeling. Calcif Tissue Res 1969;3(3):211-237.

93. Frost HM, Villanueva AR, Roth H, Stanisavljevic S. Tetracycline bone labeling. J New Drugs 1961;1:206-216.

94. Milch RA, Rall DP, Tobie JE. Bone localization of the tetracyclines. J Natl Cancer Inst 1957;19(1):87-93.

95. Pautke C, Bauer F, Tischer T, et al. Fluorescence-guided bone resection in bisphosphonate-associated osteonecrosis of the jaws. J Oral Maxillofac Surg 2009;67(3):471-476.

96. Pautke C, Tischer T, Neff A, Horch HH, Kolk A. In vivo tetracycline labeling of bone: an intraoperative aid in the surgical therapy of osteoradionecrosis of the mandible. Oral Surg Oral Med Oral Pathol Oral Radiol Endod 2006;102(6):e10-e13.

97. Celli JP, Spring BQ, Rizvi I, et al. Imaging and photodynamic therapy: mechanisms, monitoring, and optimization. Chem Rev 2010;110(5):2795-2838.

98. Santos Cortes JA, Gahan J, Soloway MS. Photodynamic diagnosis in urology: state of the art. Arch Esp Urol 2011;64(1):18-31.

99. Fotinos N, Campo MA, Popowycz F, Gurny R, Lange N. 5-Aminolevulinic acid derivatives in photomedicine: Characteristics, application and perspectives. Photochem Photobiol 2006;82(4):994-1015.

100. Stummer W, Pichlmeier U, Meinel T, et al. Fluorescence-guided surgery with 5-aminolevulinic acid for resection of malignant glioma: a randomised controlled multicentre phase III trial. Lancet Oncol 2006;7(5): 392-401.

101. Lerner SP, Liu H, Wu MF, Thomas YK, Witjes JA. Fluorescence and white light cystoscopy for detection of carcinoma in situ of the urinary bladder. Urol Oncol 2012;30(3):285-289. 
102. Zimmermann A, Ritsch-Marte M, Kostron H. mTHPC-mediated photodynamic diagnosis of malignant brain tumors. Photochem Photobiol 2001;74(4):611-616.

103. Olivo M, Fu CY, Raghavan V, Lau WK. New frontier in hypericin-mediated diagnosis of cancer with current optical technologies. Ann Biomed Eng 2012;40(2):460-473.

104. Ishizawa T, Fukushima N, Shibahara J, et al. Real-time identification of liver cancers by using indocyanine green fluorescent imaging. Cancer 2009;115(11):2491-2504.

105. Azhdarinia A, Ghosh P, Ghosh S, Wilganowski N, Sevick-Muraca EM. Dual-labeling strategies for nuclear and fluorescence molecular imaging: a review and analysis. Mol Imaging Biol 2012;14(3):261-276.

106. Guo K, Berezin MY, Zheng J, et al. Near infrared-fluorescent and magnetic resonance imaging molecular probe with high T1 relaxivity for in vivo multimodal imaging. Chem Commun (Camb) 2010;46(21):3705-3707.

107. Russell RW, Ffytche TJ, Sanders MD. A study of retinal vascular occlusion using fluorescein angiography. Lancet 1966;2 (7468):821-825.

108. Sjoback R, Nygren J, Kubista M. Absorption and fluorescence properties of fluorescein. Spectrochim Acta [A] 1995;51(6):L7-L21.

109. Haynes WF Jr, Pittman FE. Application of the fluorescein string test in 32 cases of upper gastrointestinal hemorrhage. Preliminary report. Gastroenterology 1960; 38:690-697.

110. Isak SJ, Eyring EM. Fluorescence quantum yield of cresyl violet in methanol and water as a function of concentration. J Phys Chem 1992;96(4):1738-1742.

111. Tardivo JP, Del Giglio A, de Oliveira CS, et al. Methylene blue in photodynamic therapy: From basic mechanisms to clinical applications. Photodiagn Photodyn Ther 2005; 2(3):175-191.

112. Tannous Z, Al-Arashi M, Shah S, Yaroslavsky AN. Delineating melanoma using multimodal polarized light imaging. Lasers Surg Med 2009;41(1):10-16.

113. Holm C, Tegeler J, Mayr M, Becker A, Pfeiffer UJ, Mühlbauer W. Monitoring free flaps using laser-induced fluorescence of indocyanine green: a preliminary experience. Microsurgery 2002;22(7):278-287.

114. Yuan B, Chen N, Zhu Q. Emission and absorption properties of indocyanine green in Intralipid solution. J Biomed Opt 2004;9(3):497-503.
115. Guyer DR, Yannuzzi LA, Slakter JS, et al. Classification of choroidal neovascularization by digital indocyanine green videoangiography. Ophthalmology 1996;103(12): 2054-2060.

116. Unno N, Inuzuka K, Suzuki M, et al. Pre liminary experience with a novel fluorescence lymphography using indocyanine green in patients with secondary lymphedema. J Vasc Surg 2007;45(5):1016-1021.

117. Lee Z, Simhan J, Parker DC, et al. Novel use of indocyanine green for intraoperative, real-time localization of ureteral stenosis during robot-assisted ureteroureterostomy. Urology 2013;82(3):729-733.

118. Ishizawa T, Zuker NB, Kokudo N, Gayet B. Positive and negative staining of hepatic segments by use of fluorescent imaging techniques during laparoscopic hepatectomy. Arch Surg 2012;147(4):393-394.

119. Folli $\mathrm{S}$, Wagnières G, Pèlegrin A, et al. Immunophotodiagnosis of colon carcinomas in patients injected with fluoresceinated chimeric antibodies against carcinoembryonic antigen. Proc Natl Acad Sci U S A 1992;89(17):7973-7977.

120. Mujumdar RB, Ernst LA, Mujumdar SR, Lewis CJ, Waggoner AS. Cyanine dye labeling reagents: sulfoindocyanine succinimidyl esters. Bioconjug Chem 1993;4(2):105-111.

121. Sakabe M, Asanuma D, Kamiya M, et al. Rational design of highly sensitive fluorescence probes for protease and glycosidase based on precisely controlled spirocyclization. J Am Chem Soc 2013;135(1):409-414.

122. Li Z, Zuo XL, Li CQ, et al. In vivo molecular imaging of gastric cancer by targeting MG7 antigen with confocal laser endomicroscopy. Endoscopy 2013;45(2):79-85.

123. http://clinicaltrials.gov/ct2/show/ NCT01508572.

124. Tanaka E, Choi HS, Humblet V, Ohnishi S, Laurence RG, Frangioni JV. Real-time intraoperative assessment of the extrahepatic bile ducts in rats and pigs using invisible near-infrared fluorescent light. Surgery 2008;144(1):39-48.

125. Ohnishi S, Garfein ES, Karp SJ, Frangioni JV. Radiolabeled and near-infrared fluorescent fibrinogen derivatives create a system for the identification and repair of obscure gastrointestinal bleeding. Surgery 2006;140(5) :785-792.

126. Pautke C, Bauer F, Otto S, et al. Fluorescence-guided bone resection in bisphosphonate-related osteonecrosis of the jaws: first clinical results of a prospective pilot study. J Oral Maxillofac Surg 2011;69(1):84-91 .

127. Carlotti B, Fuoco D, Elisei F. Fast and ultrafast spectroscopic investigation of tetracycline derivatives in organic and aqueous media. Phys Chem Chem Phys 2010;12(48): 15580-15591.

128. Ayala AG, Murray JA, Erling MA, Raymond AK. Osteoid-osteoma: intraoperative tetracycline-fluorescence demonstration of the nidus. J Bone Joint Surg Am 1986;68(5): 747-751.

129. Yaroslavsky AN, Salomatina EV, Neel V, Anderson R, Flotte T. Fluorescence polarization of tetracycline derivatives as a technique for mapping nonmelanoma skin cancers. J Biomed Opt 2007;12(1):014005.

130. Lozovaya GI, Masinovsky Z, Sivash AA. Protoporphyrin-Ix as a possible ancient photosensitizer - spectral and photochemical studies. Orig Life Evol Biosph 1990; 20(3-4):321-330.

131. Niziolek M, Korytowski W, Girotti AW. Selfsensitized photodegradation of membranebound protoporphyrin mediated by chain lipid peroxidation: inhibition by nitric oxide with sustained singlet oxygen damage. Photochem Photobiol 2005;81(2):299-305.

132. Yamazaki T, Ohta N, Yamazaki I, Song PS. Excited-state properties of hypericin: electronic spectra and fluorescence decay kinetics. J Phys Chem 1993;97(30):78707875.

133. García-Díaz M, Sánchez-García D, Soriano $\mathrm{J}$, et al. Temocene: the porphycene analogue of temoporfin (Foscan ${ }^{\circledR}$ ). Med Chem Commun 2011;2(7):616-619.

134. Xie H, Liu H, Svenmarker P, et al. Drug quantification in turbid media by fluorescence imaging combined with lightabsorption correction using white Monte Carlo simulations. J Biomed Opt 2011; 16(6):066002.

135. Ishizawa T, Masuda K, Satou S, et al. Indocyanine green-fluorescent imaging for identifying hepatocellular carcinoma during liver resection. From: Proceedings of the 2010 World Molecular Imaging Congress, Kyoto, Japan. Mol Imaging Biol 2010;12(2 Suppl):500-1636.

136. Lapini A, Minervini A, Masala A, et al. A comparison of hexaminolevulinate (Hex$\operatorname{vix}(\circledast))$ fluorescence cystoscopy and whitelight cystoscopy for detection of bladder cancer: results of the HeRo observational study. Surg Endosc 2012;26(12):3634-3641 